\title{
R-charge conservation and more in factorizable and non-factorizable orbifolds
}

\author{
Nana G. Cabo Bizet, ${ }^{a, b, c}$ Tatsuo Kobayashi, ${ }^{d}$ Damián K. Mayorga Peña, ${ }^{a}$ \\ Susha L. Parameswaran, ${ }^{e}$ Matthias Schmitz ${ }^{a}$ and Ivonne Zavala ${ }^{f}$ \\ ${ }^{a}$ Bethe Center for Theoretical Physics and Physikalisches Institut der Universität Bonn, \\ Nussallee 12, 53115 Bonn, Germany \\ ${ }^{b}$ Centro de Aplicaciones Tecnológicas y Desarrollo Nuclear, \\ Calle 30, esq.a 5ta Ave, Miramar, 6122 La Habana, Cuba \\ ${ }^{c}$ Theory Group, Physics Department, \\ CERN CH-1211, Genève 23, Switzerland \\ ${ }^{d}$ Department of Physics, Kyoto University, \\ Kyoto 606-8502, Japan \\ ${ }^{e}$ Department of Mathematics and Physics, Leibniz Universität Hannover, \\ Welfengarten 1, 30167 Hannover, Germany \\ ${ }^{f}$ Centre for Theoretical Physics, University of Groningen, \\ Nijenborgh 4, 9747 AG Groningen, The Netherlands \\ E-mail: nana@th.physik.uni-bonn.de, \\ kobayash@gauge.scphys.kyoto-u.ac.jp, damian@th.physik.uni-bonn.de, \\ susha.parameswaran@itp.uni-hannover.de, \\ mschmitz@th.physik.uni-bonn.de, e.i.zavala@rug.nl
}

AbstRACT: We consider the string theory origin of R-charge conservation laws in heterotic orbifold compactifications, deriving the corresponding string coupling selection rule for factorizable and non-factorizable orbifolds, with prime ordered and non-prime ordered point groups. R-charge conservation arises due to symmetries among the worldsheet instantons that can mediate the couplings. Among our results is a previously missed non-trivial contribution to the conserved R-charges from the $\gamma$-phases in non-prime orbifolds, which weakens the R-charge selection rule. Symmetries among the worldsheet instantons can also lead to additional selection rules for some couplings. We make a similar analysis for Rule 4 or the "torus lattice selection rule". Moreover, we identify a new string selection rule, that we call Rule 6 or the "coset vector selection rule".

KEYwords: Superstrings and Heterotic Strings, Conformal Field Models in String Theory, String Field Theory

ARXIV EPRINT: 1301.2322 


\section{Contents}

1 Introduction 1

2 Orbifold CFT review 2

2.1 Orbifold geometry, the space group and conjugacy classes 3

2.2 Vertex operators and correlation functions 4

2.3 String selection rules 6

3 R-charge conservation, the $\gamma$-phase and a new stringy rule 10

$\begin{array}{ll}3.1 \text { Orbifold automorphisms } & 10\end{array}$

$\begin{array}{lll}3.2 & \text { A 2D illustrative example } & 13\end{array}$

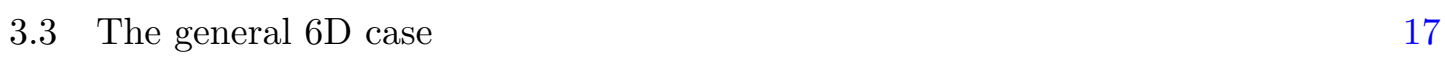

$\begin{array}{lll}3.3 .1 & \text { Factorizable orbifolds } & 18\end{array}$

$\begin{array}{lll}\text { 3.3.2 Non-factorizable orbifolds } & 22\end{array}$

4 Rule 4 in factorizable and non-factorizable orbifolds 24

$\begin{array}{llr}5 & \text { Conclusions } & 26\end{array}$

$\begin{array}{ll}\text { A A classification of orbifold automorphisms } & 31\end{array}$

\section{Introduction}

In recent years remarkable progress has been made in the quest for a string theoretic description of the standard model of particle physics. In particular we now have hundreds of explicit models whose low energy matter spectra are potentially realistic - containing the standard model particles and no chiral exotics - in several classes of string constructions; heterotic smooth Calabi-Yau compactifications [1], heterotic orbifold compactifications [2], Gepner models [3], free fermionic constructions [4], D-brane models [5], F-theory [6] (see [710] for some recent reviews). It is now essential to go beyond the particle content, and consider the low energy effective field theory that describes their phenomenology. Toroidal orbifold compactifications are attractive in this respect, as the corresponding action can at least in principle be computed explicitly, via the string CFT [11, 12], which is free.

A first question one asks is which couplings in the action are allowed to be nonvanishing. The topic of selection rules for non-vanishing superpotential couplings in heterotic orbifold compactifications was revisited recently in [13]. By studying the corresponding L-point correlation functions, an apparently forgotten rule [14-16] was identified, and a new rule discovered. These Rules 4 and 5 arise from the properties of the worldsheet instantons that can mediate couplings. 
We seek in the present paper to understand the stringy nature of R-symmetries in heterotic orbifold compactifications. It has generally been assumed that this issue is well understood for factorizable orbifolds $[17,18]$, but not understood at all for non-factorizable orbifolds $[19,20]$. We provide a derivation of the R-charge conservation law from the orbifold CFT, for factorizable and non-factorizable orbifolds, with prime ordered and nonprime ordered twists. We restrict our present analysis to compactifications without discrete Wilson lines. This is because the effect of discrete Wilson lines on the string couplings has not yet been worked out, since a consistent action description in this case is not known [21-23].

We show that the R-charge conservation law also emerges due to properties of the worldsheet instantons, with symmetries in the orbifold geometry leading to symmetries amongst the worldsheet instantons wrapping the orbifold space. For non-factorizable orbifolds, we find charge conservation laws which correspond to non-R symmetries, as well as some R-charge selection rules. At the same time, we also encounter a new stringy rule, which we may call Rule 6 , which applies in some orbifolds to some couplings. Its origin is similar to that of charge conservation and Rule 4, arising due to symmetries among worldsheet instantons.

Our results on the R-charge conservation agree with the current literature for the prime factorizable orbifolds, but for the non-prime case we find a non-trivial contribution to the conserved R-charges from the $\gamma$-phase, which has previously been unnoted. As a consequence, some couplings that are forbidden by the old R-charge conservation are actually non-vanishing. This is particularly important, as a favourite choice for Orbifolders constructing potentially realistic orbifold compactifications $[2,18,24-30]$ has been nonprime orbifolds like $T^{6} / \mathbb{Z}_{6-I I}$. Another observation that differs from the current picture is that factorizable orbifolds do not always lead to three independent R-charge conservation laws, with one associated to each plane. In fact, planes for which the orbifold twist is non-prime turn out to all contribute to a single R-charge conservation law.

The paper is organized as follows. In section 2 , we provide a brief review of the orbifold setup and outline how string selection rules can be derived from the string correlation functions. We build upon this discussion in section 3 , first considering the discrete Lorentz symmetries enjoyed by the orbifold geometry, and then deriving directly from the CFT the charge conservation laws and Rule 6 for factorizable and non-factorizable orbifolds. In section 4 , we turn to Rule 4. Finally, in section 5 , we provide a summary of our results, and discuss their significance. In an appendix we give a more detailed classification of orbifold automorphisms, for future reference.

\section{Orbifold CFT review}

Let us begin by briefly describing the orbifold geometry and associated conformal field theory [11, 12], which provides the setting for our discussion. In this section, we introduce the orbifold and the string states that emerge when heterotic string theory is compactified on the orbifold. Then we describe the basic ingredients of the corresponding worldsheet conformal field theory, in particular the vertex operators and correlation functions. Finally, we review how the correlation functions can be used to derive string coupling selection rules, referring to [13] for more details. 


\begin{tabular}{|c|c|}
\hline Point group & Twist vector \\
\hline $\mathbb{Z}_{3}$ & $(1,1,-2) / 3$ \\
$\mathbb{Z}_{4}$ & $(1,1,-2) / 4$ \\
$\mathbb{Z}_{6-I}$ & $(1,1,-2) / 6$ \\
$\mathbb{Z}_{6-I I}$ & $(1,2,-3) / 6$ \\
$\mathbb{Z}_{7}$ & $(1,2,-3) / 7$ \\
$\mathbb{Z}_{8-I}$ & $(2,1,-3) / 8$ \\
$\mathbb{Z}_{8-I I}$ & $(1,3,-4) / 8$ \\
$\mathbb{Z}_{12-I}$ & $(4,1,-5) / 12$ \\
$\mathbb{Z}_{12-I I}$ & $(1,5,-6) / 12$ \\
\hline
\end{tabular}

Table 1. All $\mathbb{Z}_{N}$ point groups and twist vectors for 6 D orbifolds leading to $\mathcal{N}=1$ SUSY $[31,32]$.

\subsection{Orbifold geometry, the space group and conjugacy classes}

$\mathrm{A} \mathbb{Z}_{\mathrm{N}}$ orbifold is constructed by dividing $\mathbb{R}^{6}$ by a six-dimensional lattice $\Lambda$ in order to obtain a torus, and subsequently modding out by some $\mathbb{Z}_{\mathrm{N}}$ automorphism. The $\mathbb{Z}_{\mathrm{N}}$ automorphism is called the point group $P$, and defining the space group $S$ as $S=\Lambda \rtimes P$, we can thus describe the orbifold as $T^{6} / P$ or $\mathbb{R}^{6} / S[31,32]$.

We call the generator of $P$ the twist, $\theta$, and it is convenient to diagonalize it, using complex coordinates to describe the torus and writing $\theta$ as:

$$
\theta=\operatorname{diag}\left(e^{2 \pi \imath v_{1}}, e^{2 \pi \imath v_{2}}, e^{2 \pi \imath v_{3}}\right) .
$$

The vector $v=\left(v_{1}, v_{2}, v_{3}\right)$ is referred to as the twist vector. The orbifold geometry has a number of special points that are fixed under the action of the point group and torus lattice shifts. We denote with $f^{(k)}$ a point fixed under $\theta^{k}$, that is $f^{(k)}=\theta^{k} f^{(k)}+\lambda$, for some $\lambda \in \Lambda$. Thus we can associate to each fixed point a space group element $g=\left(\theta^{k}, \lambda\right)$ or $g=\left(\theta^{k},\left(1-\theta^{k}\right) f^{(k)}\right)$.

We consider compactifications of the heterotic string theory on this orbifold geometry. The requirement of $\mathcal{N}=1$ supersymmetry in four dimensions then restricts the $\mathbb{Z}_{\mathrm{N}}$ point group to nine possibilities ${ }^{1}$ listed in table 1.

We will see that the properties of the orbifold compactification depend strongly on whether the order $\mathrm{N}$ of the point group is prime or non-prime. Given a point group, several underlying torus lattices may be possible [33-36], the only requirement being that the group of lattice automorphisms contains $\mathbb{Z}_{\mathrm{N}}$. Orbifolds may then also be classed according to whether or not the underlying torus lattice factorizes into three orthogonal complex planes. If so, the orbifold is called factorizable, although this is rather a misnomer as it is only the underlying torus that is factorizable, whereas the orbifold is not $\left(T^{2} / \mathbb{Z}_{\mathrm{N}_{1}}\right) \times$ $\left(T^{2} / \mathbb{Z}_{\mathrm{N}_{2}}\right) \times\left(T^{2} / \mathbb{Z}_{\mathrm{N}_{3}}\right)$.

The string states on the orbifold satisfy closed string boundary conditions that incorporate a non-trivial local monodromy:

$$
X^{i}\left(e^{2 \pi \imath} z, e^{-2 \pi \imath} \bar{z}\right)=\left(\theta^{k} X\right)^{i}(z, \bar{z})+\lambda^{i} \equiv(g X)^{i}(z, \bar{z}),
$$

\footnotetext{
${ }^{1}$ In addition there are nine $\mathbb{Z}_{\mathrm{N}} \times \mathbb{Z}_{\mathrm{M}}$ orbifolds, for which we could make an analogous discussion. More sophisticated orbifold constructions have also been considered for instance in ref. [33].
} 
with $X^{i}, i=1,2,3$, the complex coordinates of the string in the target orbifold space, and $z, \bar{z}$ the complex worldsheet coordinates. When $k=0$ we call the states untwisted, otherwise they are twisted, in the $k$-th twisted sector. A twisted state is associated, via eq. (2.2), not just to a single space group element $g$, but with an entire conjugacy class of the space group, where $\left\{h g h^{-1} \mid h \in S\right\}$ is the conjugacy class of $g$ [11].

For prime orbifolds, the conjugacy classes are in one-to-one correspondence with the fixed points of $P$ and are given by $\left\{\left(\theta^{k}, \lambda+\left(1-\theta^{k}\right) \Lambda\right)\right\}$, with the translation part running over some coset of the torus lattice $\Lambda$. For non-prime orbifolds, it may happen that a point $f$ fixed under $\theta^{k}$ is not fixed under $\theta$. We can then consider its orbit $\mathcal{O}_{f}$ as $\left\{\theta^{r} f ; 0 \leq r<l\right\}$, for $l$ the smallest integer such that $\theta^{l} f=f+\lambda$. In other words, two points fixed under $\theta^{k}$ that are different on the torus may be connected by $\theta$, in which case both correspond to the same conjugacy class and are equivalent in the orbifold. The conjugacy classes are then given by:

$$
\bigcup_{r=0}^{l-1}\left\{\left(\theta^{k}, \theta^{r} \lambda+\left(1-\theta^{k}\right) \Lambda\right)\right\},
$$

with higher twisted sectors generically corresponding to a union of cosets. It follows that for non-prime orbifolds, the physical states in the $k$-th twisted sector are in general linear combinations of states located at several $\theta^{k}$ fixed points [35-37]. Labelling states by their associated fixed points we construct the physical states $|\psi\rangle$ as

$$
|\psi\rangle=|f\rangle+e^{-2 \pi \imath \gamma}|\theta f\rangle+\cdots+e^{-2 \pi \imath(l-1) \gamma}\left|\theta^{l-1} f\right\rangle,
$$

with the possible $\gamma$-phases given by $\gamma=p / l, p=0,1, \ldots, l-1$. The geometric part of the string state is thus constructed to be an eigenstate of the $\theta$ twist, with eigenvalue given by $e^{2 \pi \imath \gamma}$.

So far we have presented the action of the $\mathbb{Z}_{N}$ point group on the bosonic coordinate fields $X^{i}$, but modular invariance requires the twist to act non-trivially on the other components of the heterotic string. In particular, excluding Wilson lines, the twist is embedded into the gauge group with the gauge degrees of freedom transforming as $X^{I} \rightarrow X^{I}+2 \pi V^{I}$, $I=1, \ldots, 16$, where $V^{I}$ is called the gauge shift vector.

The physical string states must be invariant under the total $\mathbb{Z}_{\mathrm{N}}$ transformation. We will show this in more detail below, in terms of the corresponding vertex operators.

\subsection{Vertex operators and correlation functions}

The physical states in the string Hilbert space correspond to fields or operators in the orbifold CFT. To compute the low energy effective field theory, we are interested in the vertex operators describing the emission of massless fields, in the limit of zero $4 \mathrm{D}$ momentum. For a twisted bosonic field, this is given by: ${ }^{2}$

$$
V_{-1}=e^{-\phi} \prod_{i=1}^{3}\left(\partial X^{i}\right)^{\mathcal{N}_{L}^{i}}\left(\partial \bar{X}^{i}\right)^{\overline{\mathcal{N}}_{L}^{i}} e^{\imath q_{s h}^{m} H^{m}} e^{\imath p_{s h}^{I} X^{I}} \sigma_{(k, \psi)}^{i},
$$

\footnotetext{
${ }^{2}$ We omit normalization and cocycle factors, as they are not important for our purposes.
} 
whilst for a twisted fermionic field it is

$$
V_{-1 / 2}=e^{-\phi / 2} \prod_{i=1}^{3}\left(\partial X^{i}\right)^{\mathcal{N}_{L}^{i}}\left(\partial \bar{X}^{i}\right)^{\overline{\mathcal{N}}_{L}^{i}} e^{\imath q_{s h}^{(f) m} H^{m}} e^{\imath p_{s h}^{I} X^{I}} \sigma_{(k, \psi)}^{i} .
$$

Each has a number of contributions, corresponding to the various degrees of freedom carried by the heterotic string. The right-moving worldsheet fermions have been bosonized to the fields $H^{m}(\bar{z}), m=1, \ldots, 5$, and they carry an H-momentum given by $q_{s h}=q+k v$ for the twisted spacetime bosonic states, with $q$ a weight on the vector lattice of $\mathrm{SO}(10)$, and $k v$ the shift induced by the orbifold twist. The H-momentum for the spinor representation is written as $q_{s h}^{(f)}$, and is related to that in the vector representation by:

$$
q_{s h}=q_{s h}^{(f)}+(1,1,1,1,1) / 2 .
$$

The left-moving worldsheet bosonic gauge fields correspond to $X^{I}(z), I=1, \ldots, 16$, which carry a similarly shifted gauge momentum, $p_{s h}=p+k V$, where $p$ is a vector in the $E_{8} \times E_{8}$ lattice. Massless states may be excited via some number, $\mathcal{N}_{L}^{i}, \overline{\mathcal{N}}_{L}^{i}$, of left-moving holomorphic and anti-holomorphic bosonic oscillators. We have also introduced a scalar field, the superconformal ghost $\phi$, with the subscript on $V$ indicating the superconformal ghost charge.

The twist fields $\sigma_{(k, \psi)}$ serve to implement the non-trivial monodromy (2.2) observed for the bosonic string coordinates $X^{i}(z, \bar{z})$. For the prime orbifolds there is a one-to-one correspondence between the twist fields and fixed points, but for non-prime orbifolds it is helpful to construct them as [38, 39]:

$$
\sigma_{(k, \psi)}=\sum_{r=0}^{l-1} e^{-2 \pi \imath r \gamma} \sigma_{\left(k, \theta^{r} f\right)},
$$

with $\sigma_{\left(k, \theta^{r} f\right)}$ being viewed as auxiliary twist fields. Note that, as far as quantum properties go, the auxiliary twist fields $\sigma_{\left(k, \theta^{r} f\right)}$ with various $\theta^{r} f$ are indistinguishable. Also, untwisted fields, which have $k=0$, are included in our discussion by taking $\sigma_{(k, f)}^{i} \rightarrow 1$.

The quantum numbers of the physical massless states should fulfill the masslessness conditions and orbifold projection conditions. Under the twist, the complete state associated with the space group element $g=\left(\theta^{k}, \lambda\right)$ acquires a phase:

$$
\Delta\left(k, e^{2 \pi \imath \gamma}\right)=e^{2 \pi \imath\left[\left(\mathcal{N}_{L}-\overline{\mathcal{N}}_{L}\right) \cdot v+p_{s h} \cdot V-q_{s h} \cdot v+\gamma-\frac{k}{2}\left(V^{2}-v^{2}\right)\right]},
$$

so $\Delta\left(k, e^{2 \pi \imath \gamma}\right)=1$ for physical states.

With the vertex operators at hand, we are ready to consider the correlation functions. We are interested in string tree-level L-point correlation functions of the kind $\left\langle V_{F} V_{F} V_{B} \ldots V_{B}\right\rangle$, as this is enough to identify allowed terms in the holomorphic superpotential of the low energy effective field theory. To cancel the background ghost-charge, which is 2 on the worldsheet sphere, we have to introduce $\mathrm{L}-3$ picture-changing operators 
into the correlation function, taking $\left\langle V_{-1 / 2} V_{-1 / 2} V_{-1} V_{0} \ldots V_{0}\right\rangle$ with

$$
\begin{aligned}
V_{0}=\sum_{j=1}^{3}\left(e^{\imath q_{0}^{j m}} H^{m}\right. & \left.\bar{\partial} X^{j}+e^{-\imath q_{0}^{j m} H^{m}} \bar{\partial} \bar{X}^{j}\right) \\
& \times \prod_{i=1}^{3}\left(\partial X^{i}\right)^{\mathcal{N}_{L}^{i}}\left(\partial \bar{X}^{i}\right)^{\overline{\mathcal{N}}_{L}^{i}} e^{\imath q_{s h}^{m} H^{m}} e^{\imath p_{s h}^{I} X^{I}} \sigma_{(k, \psi)}^{i} .
\end{aligned}
$$

Thus, for higher order couplings, with $\mathrm{L}>3$, additional H-momenta $q_{0}^{1}=(0,0,1,0,0)$, $q_{0}^{2}=(0,0,0,1,0)$ and $q_{0}^{3}=(0,0,0,0,1)$ need to be introduced, as well as right-moving oscillators, which we count by $\mathcal{N}_{R}^{i}, \overline{\mathcal{N}}_{R}^{i}$.

\subsection{String selection rules}

We now review how string coupling selection rules can be derived from the string correlation functions [11-13]. In particular, in this section we recall the space group selection rule, gauge invariance, H-momentum conservation and Rule 5, referring the reader to [13] for more details. In the following sections we will turn to the main topic of the paper; Rcharge conservation and a new stringy rule, as well as Rule 4, in factorizable and nonfactorizable orbifolds.

Since the orbifold CFT is free, the L-point correlation functions factorize into several parts, each giving rise to a certain condition for non-vanishing couplings. The twist fields lead to the so-called space group selection rule, which takes the form $(\alpha=1, \ldots, \mathrm{L})$ :

$$
\prod_{\alpha=1}^{\mathrm{L}}\left[g_{\alpha}\right]=(\mathbb{1}, 0),
$$

where $\left[g_{\alpha}\right]$ represents some element of the conjugacy class of the space group element $g_{\alpha}$. The space group selection rule includes the point group selection rule, $\sum_{\alpha=1}^{\mathrm{L}} k_{\alpha}=0 \bmod \mathrm{N}$ for the $\mathbb{Z}_{\mathrm{N}}$ orbifold. In terms of the explicit space group elements, it can be written as ${ }^{3}$

$$
\begin{gathered}
\left(1-\theta^{k_{\mathrm{L}}}\right)\left(\theta^{r_{\mathrm{L}}} f_{\mathrm{L}}+\tau_{\mathrm{L}}\right)+\theta^{k_{\mathrm{L}}}\left(1-\theta^{k_{\mathrm{L}-1}}\right)\left(\theta^{r_{\mathrm{L}-1}} f_{\mathrm{L}-1}+\tau_{\mathrm{L}-1}\right)+\ldots \\
\ldots+\theta^{k_{\mathrm{L}}+k_{\mathrm{L}-1}+\cdots+k_{2}}\left(1-\theta^{k_{1}}\right)\left(\theta^{r_{1}} f_{1}+\tau_{1}\right)=0,
\end{gathered}
$$

for some integers $0 \leq r_{\alpha}<\mathrm{N}$ and some $\tau_{\alpha} \in \Lambda$. Thus we see that the space group selection rule restricts the combinations of fixed points that can enter a coupling.

Next we have the momentum conservation conditions. The conservation of gauge momentum:

$$
\sum_{\alpha} p_{s h}=0
$$

leads to gauge invariance. Instead, conservation of H-momentum implies that all terms in the correlation function vanish except for those satisfying:

$$
\mathcal{N}_{R}^{i}=0, \quad \overline{\mathcal{N}}_{R}^{i}=\sum_{\alpha} q_{s h \alpha}^{i}-1 .
$$

\footnotetext{
${ }^{3}$ In a slight abuse of notation, throughout the text we use $\theta$ to denote both the generator of $P$ and the Coxeter element of the lattice Lie algebra, which gives the action of the point group on the basis vectors of the lattice. The meaning should however be clear from the context.
} 
Note that for higher order couplings, H-momentum conservation is automatically satisfied after imposing the point group selection rule, but must be considered to fix the number of right-moving oscillators in the correlation function. We will comment on H-momentum conservation for three-point couplings below.

After applying the momentum conservation, the non-trivial part of the general correlation function takes the form [13-16, 40]:

$$
\mathcal{F}=\prod_{i=1}^{3}\left\langle\left(\partial X^{i}\right)^{\mathcal{N}_{L}^{i}}\left(\partial \bar{X}^{i}\right)^{\overline{\mathcal{N}}_{L}^{i}}\left(\bar{\partial} \bar{X}^{i}\right)^{\overline{\mathcal{N}}_{R}^{i}} \sigma_{\left(k_{1}, \psi_{1}\right)}^{i} \cdots \sigma_{\left(k_{\mathrm{L}}, \psi_{\mathrm{L}}\right)}^{i}\right\rangle
$$

where we have factorized it into $2 \mathrm{D}$ components, and from now on $\mathcal{N}_{L}^{i}, \overline{\mathcal{N}}_{L}^{i}$, like $\overline{\mathcal{N}}_{R}^{i}$, refer to the total number of oscillators appearing in the correlation function. Notice moreover that for the higher twisted sectors, we can write the correlation function for physical states as a sum of several auxiliary correlation functions, each involving the auxiliary twist fields, weighted by the $\gamma$-phases $[11,41,42]$ :

$$
\mathcal{F}=\sum_{r_{1}=0}^{l_{1}} \cdots \sum_{r_{\mathrm{L}}=0}^{l_{\mathrm{L}}} e^{-2 \pi \imath r_{1} \gamma_{1}-\cdots-2 \pi \imath r_{\mathrm{L}} \gamma_{\mathrm{L}}} \times \prod_{i=1}^{3} \mathcal{F}_{\text {aux }}^{i},
$$

where

$$
\mathcal{F}_{\text {aux }}^{i}=\left\langle\left(\partial X^{i}\right)^{\mathcal{N}_{L}^{i}}\left(\partial \bar{X}^{i}\right)^{\overline{\mathcal{N}}_{L}^{i}}\left(\bar{\partial} \bar{X}^{i}\right)^{\overline{\mathcal{N}}_{R}^{i}} \sigma_{\left(k_{1}, \theta^{r} f_{1}\right)}^{i} \cdots \sigma_{\left(k_{\mathrm{L}}, \theta^{r} \mathrm{~L} f_{\mathrm{L}}\right)}^{i}\right\rangle .
$$

To make further progress, it is helpful to split the bosonic coordinates into the solutions to their classical equations of motion, $\partial \bar{\partial} X_{c l}^{i}=0$, and their quantum fluctuations:

$$
X^{i}(z, \bar{z})=X_{c l}^{i}(z, \bar{z})+X_{q u}^{i}(z, \bar{z}) .
$$

The auxiliary correlation functions similarly split as:

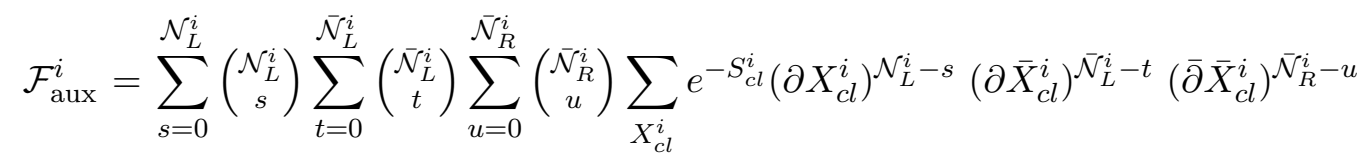

$$
\begin{aligned}
& \times \int \mathcal{D} X_{q u}^{i} e^{-S_{q u}^{i}}\left(\partial X_{q u}^{i}\right)^{s}\left(\partial \bar{X}_{q u}^{i}\right)^{t}\left(\bar{\partial} \bar{X}_{q u}^{i}\right)^{u} \sigma_{\left(k_{1}, \theta^{r} f_{1}\right)}^{i} \cdots \sigma_{\left(k_{\mathrm{L}}, \theta^{r} \mathrm{~L} f_{\mathrm{L}}\right)}^{i},
\end{aligned}
$$

where $\left(\begin{array}{c}\mathcal{N}_{L}^{i} \\ s\end{array}\right)$ and so on are the binomial coefficients and the classical action is given by

$$
S_{c l}^{i}=\frac{1}{8 \pi} \int d^{2} z\left(\left|\partial X_{c l}^{i}\right|^{2}+\left|\partial \bar{X}_{c l}^{i}\right|^{2}\right) .
$$

Moreover, for the quantum part of the correlation functions to be non-vanishing, the number of holomorphic and anti-holomorphic indices have to match [12], $s=t+u$. Thus, in fact:

$$
\begin{aligned}
& \mathcal{F}_{\text {aux }}^{i}=\sum_{t=0}^{\overline{\mathcal{N}}_{L}^{i}} \sum_{u=0}^{\overline{\mathcal{N}}_{R}^{i}}\left(\begin{array}{c}
\mathcal{N}_{L}^{i} \\
t+u
\end{array}\right)\left(\begin{array}{c}
\overline{\mathcal{N}}_{L}^{i} \\
t
\end{array}\right)\left(\begin{array}{c}
\overline{\mathcal{N}}_{R}^{i} \\
u
\end{array}\right) \sum_{X_{c l}^{i}} e^{-S_{c l}^{i}}\left(\partial X_{c l}^{i}\right)^{\mathcal{N}_{L}^{i}-t-u}\left(\partial \bar{X}_{c l}^{i}\right)^{\overline{\mathcal{N}}_{L}^{i}-t}\left(\bar{\partial} \bar{X}_{c l}^{i}\right)^{\overline{\mathcal{N}}_{R}^{i}-u} \\
& \times \int \mathcal{D} X_{q u}^{i} e^{-S_{q u}^{i}}\left(\partial X_{q u}^{i}\right)^{t+u}\left(\partial \bar{X}_{q u}^{i}\right)^{t}\left(\bar{\partial} \bar{X}_{q u}^{i}\right)^{u} \sigma_{\left(k_{1}, \theta^{r} 1 f_{1}\right)}^{i} \cdots \sigma_{\left(k_{\mathrm{L}}, \theta^{r} \mathrm{~L} f_{\mathrm{L}}\right)}^{i},
\end{aligned}
$$

where the non-zero contributions in the sums over $t$ and $u$ satisfy $t+u \leq \mathcal{N}_{L}^{i}$. 
The classical solutions represent worldsheet instantons stretching between the fixed points involved in the auxiliary couplings, and can be written as [11, 41-44]:

$$
\begin{aligned}
& \partial X_{c l}^{i}(z)=\sum_{l=1}^{\mathrm{L}-\mathrm{M}^{i}-1} a_{l}^{i} h_{l}^{i}(z), \\
& \bar{\partial} X_{c l}^{i}(\bar{z})=\sum_{l^{\prime}=1}^{\mathrm{M}^{i}-1} b_{l^{\prime}}^{i}{\overline{h^{\prime}}}_{l^{\prime}}^{i}(\bar{z}),
\end{aligned}
$$

(no summation over $i$ ), plus their complex conjugates:

$$
\begin{aligned}
\bar{\partial} \bar{X}_{c l}^{i} & =\left(\partial X_{c l}^{i}\right)^{*}, \\
\bar{\partial} X_{c l}^{i} & =\left(\partial \bar{X}_{c l}^{i}\right)^{*} .
\end{aligned}
$$

Here, the integers $\mathrm{M}^{i}$ are given by $\mathrm{M}^{i}=\sum_{\alpha=1}^{\mathrm{L}} \mathbf{k}_{\alpha}^{i}$, and we have defined $\mathbf{k}_{\alpha}^{i}=k_{\alpha} v^{i} \bmod 1$, such that $0<\mathbf{k}_{\alpha}^{i} \leq 1$ in (2.22), and $0 \leq \mathbf{k}_{\alpha}^{i}<1$ in (2.23). Any untwisted strings present in the coupling do not alter these instanton solutions.

The basis functions $h_{l}^{i}(z),{\overline{h^{\prime}}}_{l^{\prime}}^{i}(\bar{z})$ are determined by the local monodromy conditions (2.2), and the requirement that the classical action converges [43, 44]. It can happen that the only way to satisfy these conditions is with a vanishing solution $\partial X_{c l}^{i}=0$ or $\partial \bar{X}_{c l}^{i}=0$. In particular, at least three twisted strings in a given plane are required for a non-trivial worldsheet instanton to exist in that plane. This implies Rule 5 or the forbidden instanton rule, which imposes the following conditions on the oscillator numbers [13]:

$$
\begin{aligned}
& \text { holomorphic instantons forbidden: } \mathcal{N}_{L}^{i} \leq \overline{\mathcal{N}}_{L}^{i}+\overline{\mathcal{N}}_{R}^{i} \\
& \text { anti-holomorphic instantons forbidden: } \mathcal{N}_{L}^{i} \geq \overline{\mathcal{N}}_{L}^{i} \\
& \text { no instantons allowed: } \mathcal{N}_{L}^{i}=\overline{\mathcal{N}}_{L}^{i}+\overline{\mathcal{N}}_{R}^{i},
\end{aligned}
$$

and simplifies further the expression (2.21) for the correlation function. In detail, for holomorphic instantons forbidden, the auxiliary correlation functions in the $i$-th plane take the form:

$$
\begin{aligned}
\mathcal{F}_{\text {aux }}^{i}=\left(\begin{array}{c}
\overline{\mathcal{N}}_{L}^{i} \\
\mathcal{N}_{L}^{i}-\overline{\mathcal{N}}_{R}^{i}
\end{array}\right) \sum_{X_{c l}^{i}} e^{-S_{c l}^{i}\left(\partial \bar{X}_{c l}^{i}\right)^{\overline{\mathcal{N}}_{L}^{i}-\mathcal{N}_{L}^{i}+\overline{\mathcal{N}}_{R}^{i}}} \\
\quad \times \int \mathcal{D} X_{q u}^{i} e^{-S_{q u}^{i}}\left(\partial X_{q u}^{i}\right)^{\mathcal{N}_{L}^{i}}\left(\partial \bar{X}_{q u}^{i}\right)^{\mathcal{N}_{L}^{i}-\overline{\mathcal{N}}_{R}^{i}}\left(\bar{\partial} \bar{X}_{q u}^{i}\right)^{\overline{\mathcal{N}}_{R}^{i}} \sigma_{\left(k_{1}, \theta^{r} f_{1}\right)}^{i} \cdots \sigma_{\left(k_{\mathrm{L}}, \theta^{r} \mathrm{~L} f_{\mathrm{L}}\right)}^{i}
\end{aligned}
$$

for anti-holomorphic instantons forbidden, we have:

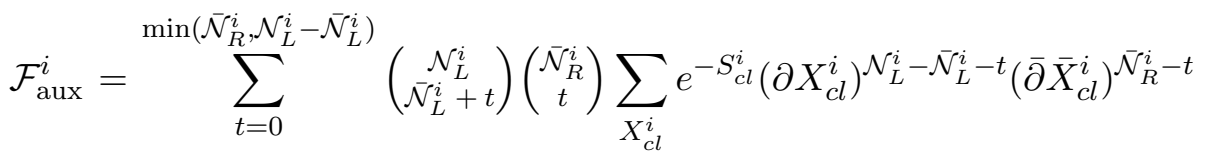

$$
\begin{aligned}
& \times \int \mathcal{D} X_{q u}^{i} e^{-S_{q u}^{i}}\left(\partial X_{q u}^{i}\right)^{\overline{\mathcal{N}}_{L}^{i}+t}\left(\partial \bar{X}_{q u}^{i}\right)^{\overline{\mathcal{N}}_{L}^{i}}\left(\bar{\partial} \bar{X}_{q u}^{i}\right)^{t} \sigma_{\left(k_{1}, \theta^{r} f_{1}\right)}^{i} \cdots \sigma_{\left(k_{\mathrm{L}}, \theta^{r} \mathrm{~L} f_{\mathrm{L}}\right)}^{i},
\end{aligned}
$$

and for no instantons allowed:

$$
\mathcal{F}_{\text {aux }}^{i}=\int \mathcal{D} X_{q u}^{i} e^{-S_{q u}^{i}}\left(\partial X_{q u}^{i}\right)^{\mathcal{N}_{L}^{i}}\left(\partial \bar{X}_{q u}^{i}\right)^{\overline{\mathcal{N}}_{L}^{i}}\left(\bar{\partial} \bar{X}_{q u}^{i}\right)^{\overline{\mathcal{N}}_{R}^{i}} \sigma_{\left(k_{1}, \theta^{\left.r_{1} f_{1}\right)}\right.}^{i} \cdots \sigma_{\left(k_{\mathrm{L}}, \theta^{r} \mathrm{~L} f_{\mathrm{L}}\right)}^{i} .
$$


To obtain further selection rules, the coefficients $a_{l}, b_{l^{\prime}}$ in the worldsheet instanton solutions will be important. These are determined by the global monodromy conditions (the quantum part instead feels only the local monodromy) [11, 41, 42, 45, 46]:

$$
\begin{gathered}
\int_{\Gamma_{p}} d z \partial X_{c l}^{i}+\int_{\Gamma_{p}} d \bar{z} \bar{\partial} X_{c l}^{i}=\nu_{p}^{i} \\
\int_{\Gamma_{p}} d z \partial \bar{X}_{c l}^{i}+\int_{\Gamma_{p}} d \bar{z} \bar{\partial} \bar{X}_{c l}^{i}=\bar{\nu}_{p}^{i},
\end{gathered}
$$

where $\Gamma_{p}$ represent all possible net zero-twist closed loops enclosing the twist fields. There will be L-2 independent such loops, which we can take to be those encircling the fixed point $f_{p}$ clockwise $n_{p}$ times followed by the fixed point $f_{p+1}$ counterclockwise $m_{p}$ times, where $n_{p} k_{p}=m_{p} k_{p+1} \bmod \mathrm{N}$, with $n_{p}, m_{p}$ the smallest integers satisfying this property, and $p=1, \ldots, \mathrm{L}-2$. The vectors $\nu_{p}^{i}$ are then elements of the corresponding cosets of the torus lattice:

$$
\nu_{p}=\left(1-\theta^{n_{p} k_{p}}\right)\left(f_{p+1}-f_{p}+\lambda\right), \quad \lambda \in \Lambda .
$$

Note that the $(\mathrm{L}-1)$-th loop is not independent from the others, as the sum of all $\mathrm{L}-1$ loops can be pulled around the worldsheet sphere and shrunk to zero, giving the space group selection rule. However, this provides a consistency constraint that may further restrict the coset vectors $\nu_{p}^{i}$. For example, for the 3-point couplings it turns out that the coset vectors are restricted to [41, 42]:

$$
\nu_{1}=\left(1-\theta^{n_{1} k_{1}}\right)\left(f_{2}-f_{1}-\tau_{2}+\tau_{1}+\left(1-\theta^{k_{1}+k_{2}}\right)\left(1-\theta^{\operatorname{gcd}\left(k_{1}, k_{2}\right)}\right)^{-1} \lambda\right), \quad \lambda \in \Lambda,
$$

where $\tau_{1,2}$ are the torus lattice vectors that appear in the space group selection rule (2.12) and gcd stands for greatest common divisor. Finally, we can solve the global monodromy conditions for the coefficients $a_{l}^{i}, b_{l^{\prime}}^{i}$. Defining the period matrices as:

$$
\begin{aligned}
W_{p}^{i l} & =\int_{\Gamma_{p}} d z h_{l}^{i}(z), & l & =1, \ldots, \mathrm{L}-\mathrm{M}^{i}-1, \\
W_{p}^{i\left(\mathrm{~L}-\mathrm{M}^{i}-1+l^{\prime}\right)} & =\int_{\Gamma_{p}} d \bar{z}{\overline{h^{\prime}}}_{l^{\prime}}^{i}(\bar{z}), & & l^{\prime}=1, \ldots, \mathrm{M}^{i}-1,
\end{aligned}
$$

it follows that $a_{l}^{i}, b_{l^{\prime}}^{i}$ are particular linear combinations of the coset vectors $\left\{\nu_{p}^{i}\right\}$ :

$$
\begin{aligned}
& a_{l}^{i}=\nu_{p}^{i}\left(W^{-1}\right)_{l}^{i p}, \\
& b_{l^{\prime}}^{i}=\nu_{p}^{i}\left(W^{-1}\right)_{L-M^{i}-1+l^{\prime}}^{i p}
\end{aligned}
$$

Notice that the fixed point dependence lies only in the coset vectors $\nu_{p}^{i}$, as the period matrices depend only on the twisted sectors involved.

We are now ready to derive more selection rules, which arise due to the symmetries amongst the worldsheet instanton solutions. To this purpose, we use eqs. (2.21) and (2.27) to show that, when holomorphic instantons are allowed in the $i$-th plane, the auxiliary correlation functions can always be written in the form:

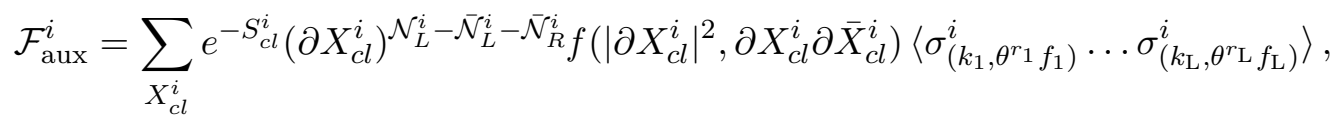


where the explicit expression for $f\left(\left|\partial X_{c l}\right|^{2}, \partial X_{c l}^{i} \partial \bar{X}_{c l}^{i}\right)$ follows from eqs. (2.21) and (2.27), and depends on the classical solutions as written, and we use the shorthand

$\left\langle\sigma_{\theta^{r} 1 f_{1}}^{i} \ldots \sigma_{\theta^{r} \mathrm{~L} f_{\mathrm{L}}}^{i}\right\rangle$ for the quantum part of the correlation function. Similarly, when holomorphic instantons are forbidden in the $i$-th plane, it follows from eqs. (2.26) and (2.28) that we can write the auxiliary correlation functions as:

$$
\mathcal{F}_{\text {aux }}^{i}=\sum_{X_{c l}^{i}} e^{-S_{c l}^{i}}\left(\partial \bar{X}_{c l}^{i}\right)^{-\mathcal{N}_{L}^{i}+\overline{\mathcal{N}}_{L}^{i}+\overline{\mathcal{N}}_{R}^{i}}\left\langle\sigma_{\left(k_{1}, \theta^{r} 1 f_{1}\right)}^{i} \ldots \sigma_{\left(k_{\mathrm{L}}, \theta^{r} \mathrm{~L} f_{\mathrm{L}}\right)}^{i}\right\rangle .
$$

It is important to observe that the classical solutions depend on the fixed points to which the participating auxiliary twist fields are associated. In detail, since the basis functions $h_{l}^{i}, h_{l^{\prime}}^{i^{\prime}}$ are determined by the local monodromy, they depend only on the twisted sectors involved in the coupling, and not the fixed points. Instead, the coefficients $a_{l}^{i}, b_{l^{\prime}}^{i}$ are determined by the global monodromy, and so do depend on the fixed point positions. If the fixed points involved in two couplings are related by the orbifold twist, then so will be the coefficients $a_{l}^{i}, b_{l^{\prime}}^{i}$ and hence also the classical solutions. Notice also that given two classical solutions, $\partial X_{c l 1}^{i}, \partial X_{c l 2}^{i}$ related by $\theta$, then $\left|\partial X_{c l 1}^{i}\right|^{2}=\left|\partial X_{c l 2}^{i}\right|^{2}$ and $\partial X_{c l 1}^{i} \partial \bar{X}_{c l 1}^{i}=\partial X_{c l 2}^{i} \partial \bar{X}_{c l 2}^{i}$.

\section{R-charge conservation, the $\gamma$-phase and a new stringy rule}

Our purpose is now to use what we have learned to study the origin and structure of the $\mathrm{R}$-charge conservation laws. R-charge conservation in orbifold compactifications has been understood from two related perspectives [14-18]. Since Lorentz symmetries distinguish between bosons and fermions, one expects any discrete Lorentz symmetries in the internal space that survive the orbifold compactification to lead to a discrete R-charge conservation law in the four dimensional effective field theory. On the more technical side, the R-charge conservation law has been derived from the general structure of the string correlation functions, as a consequence of the H-momentum conservation condition and the plane by plane twist invariance that is observed in factorizable prime orbifolds [13].

We now refine these arguments, considering carefully the various classes of $\mathbb{Z}_{\mathrm{N}}$ orbifolds, prime, non-prime, factorizable, non-factorizable. In doing so, we find that the relation between Lorentz symmetries in the orbifold geometry and R-symmetries in the low energy effective field theory can be more subtle than previously assumed. Also, the R-charges of higher twisted states in non-prime orbifolds have a non-trivial contribution from their $\gamma$-phase. Moreover, for certain orbifolds we find an additional selection rule whose string origin is similar to that of the R-charge conservation law. This rule, which we may call Rule 6 , depends however on the relative properties of the states in a coupling, and hence does not have a simple field theoretical interpretation.

\subsection{Orbifold automorphisms}

To begin with, we are interested in the symmetries of the internal space of the string compactification, that is the orbifold $T^{6} / \mathbb{Z}_{\mathrm{N}}$. Thus we are looking for the subgroup of the torus lattice automorphism group that respects the point group and moreover leaves the conjugacy classes of the fixed points invariant. We focus on the Lorentz transformations 
that are discrete rotations. In the appendix, we discuss more generally the symmetries of the orbifold geometry.

When the underlying torus lattice is factorizable, the action of the orbifold twist $\theta$ can be decomposed into discrete rotations acting plane by plane, $\left(\theta_{1}, \theta_{2}, \theta_{3}\right)$, and the fixed points can be decomposed as a direct product of fixed points in the three planes, $f^{(k)}=g_{1} \otimes g_{2} \otimes g_{3}$, with $\left(\theta_{i}\right)^{k} g_{i}=g_{i}$ (up to lattice identifications) for $f^{(k)}$ in the $k$-th twisted sector. We can then immediately identify discrete rotational symmetries. First, notice that for a plane with prime order twist, $\theta_{i} g_{i}=g_{i}$ for all the twisted sectors. Then, there are three different cases:

(i) All planes have prime order twists. In this case, all the fixed points are fixed under the orbifold twist plane by plane:

$$
\theta_{i} f^{(k)}=f^{(k)}, \quad i=1,2,3, \quad \text { for all } f^{(k)} .
$$

So we have the discrete symmetries generated by $\theta_{1}, \theta_{2}$ and $\theta_{3}$. An example of this case is $T^{6} / \mathbb{Z}_{3}$.

(ii) Only one plane is non-prime. Here, all fixed points are fixed under the prime plane rotations, say $\theta_{2}, \theta_{3}$. Moreover, considering the non-prime rotation, say $\theta_{1}$, we have:

$$
\begin{aligned}
\theta_{1} f^{(k)} & =\theta_{1} g_{1} \otimes g_{2} \otimes g_{3} \\
& =\theta_{1} g_{1} \otimes \theta_{2} g_{2} \otimes \theta_{3} g_{3} \\
& =\theta f^{(k)} \\
& \simeq f^{(k)} .
\end{aligned}
$$

where the last $\simeq$ indicates equivalence of the fixed points up to the conjugacy class. So we have again the symmetries, $\theta_{1}, \theta_{2}$ and $\theta_{3}$. Here, an example is $T^{6} / \mathbb{Z}_{6-I I}$.

(iii) Two planes are non-prime. Again, all the fixed points are fixed under the prime plane rotation, say $\theta_{3}$. Moreover, they are invariant under the combined action of the non-prime rotations, say $\theta_{1} \theta_{2}$ since:

$$
\begin{aligned}
\theta_{1} \theta_{2} f^{(k)} & =\theta_{1} g_{1} \otimes \theta_{2} g_{2} \otimes g_{3} \\
& =\theta_{1} g_{1} \otimes \theta_{2} g_{2} \otimes \theta_{3} g_{3} \\
& =\theta f^{(k)} \\
& \simeq f^{(k)} .
\end{aligned}
$$

In this case, the symmetries are generated by $\theta_{1} \theta_{2}$ and $\theta_{3}$. An example is $T^{6} / \mathbb{Z}_{6-I}$.

There is one more case to be considered. The $\mathbb{Z}_{4}$ orbifold, with twist vector $v=\frac{1}{4}(1,1,-2)$, has only two kinds of twisted sectors, $\theta, \theta^{2}$ (plus their conjugates). Therefore, all the fixed points are fixed under $\theta^{2}$, and this holds plane by plane for the factorizable orbifold:

$$
\begin{aligned}
(\theta)^{2} f^{(k)}=\left(\theta_{1}\right)^{2} g_{1} \otimes\left(\theta_{2}\right)^{2} g_{2} \otimes\left(\theta_{3}\right)^{2} g_{3}=g_{1} \otimes g_{2} \otimes g_{3}=f^{(k)} & \Rightarrow \\
\left(\theta_{1}\right)^{2} f^{(k)}=f^{(k)}, \quad\left(\theta_{2}\right)^{2} f^{(k)} & =f^{(k)} .
\end{aligned}
$$




\begin{tabular}{|c|c|c|c|}
\hline & Lattice & Twist & Orbifold Automorphisms \\
\hline $\mathbb{Z}_{4}$ & $\mathrm{SU}(4) \otimes \mathrm{SU}(4)$ & $\frac{1}{4}(1,1,-2)$ & $\theta,\left(\theta_{1}\right)^{2}$ \\
\hline $\mathbb{Z}_{6-\mathrm{II}}$ & $\mathrm{SU}(6) \otimes \mathrm{SU}(2)$ & $\frac{1}{6}(1,2,-3)$ & $\theta$ \\
\hline $\mathbb{Z}_{7}$ & $\mathrm{SU}(7)$ & $\frac{1}{7}(1,2,-3)$ & $\theta$ \\
\hline $\mathbb{Z}_{8-\mathrm{I}}$ & $\mathrm{SO}(5) \otimes \mathrm{SO}(9)$ & $\frac{1}{8}(2,1,-3)$ & $\theta,\left(\theta_{1}\right)^{2}$ \\
\hline $\mathbb{Z}_{8-\mathrm{II}}$ & $\mathrm{SO}(8) \otimes \mathrm{SO}(4)$ & $\frac{1}{8}(1,3,-4)$ & $\theta, \theta_{3}$ \\
\hline $\mathbb{Z}_{12-\mathrm{I}}$ & $\mathrm{SU}(3) \otimes F_{4}$ & $\frac{1}{12}(4,1,-5)$ & $\theta, \theta_{1}$ \\
\hline $\mathbb{Z}_{12-\mathrm{II}}$ & $F_{4} \otimes \mathrm{SO}(4)$ & $\frac{1}{12}(1,5,-6)$ & $\theta, \theta_{3}$ \\
\hline
\end{tabular}

Table 2. Orbifold automorphisms for some non-factorizable or partially factorizable orbifolds built on Lie lattices, counting independent discrete rotational symmetries that preserve the conjugacy classes, and labeling them with their generators. We refer to [35, 36] for details of the torus lattice, orbifold twist and fixed points.

Thus there will be an additional symmetry generated by $\left(\theta_{1}\right)^{2}$ or $\left(\theta_{2}\right)^{2}$ (these are not independent).

For orbifolds whose underlying torus lattice and orbifold twist are partially factorizable on one complex plane, a similar analysis can be made. For the non-factorizable orbifolds, the twist does not act plane by plane, and we cannot decompose the fixed points as a direct product of fixed points in the three planes. We may still ask what are the discrete rotational symmetries of the orbifold geometry, that is which elements of the Cartan subalgebra of $\mathrm{SO}(6)$ leave the torus lattice and fixed point conjugacy classes invariant. We have performed a computer scan for such orbifold automorphisms in several explicit examples, with results given in table 2 . In most cases, the only symmetry is the point group itself, but notice that for the $\mathbb{Z}_{4}$ and $\mathbb{Z}_{8-I}$ orbifolds, the fixed points are such that they enjoy an additional $\mathbb{Z}_{2}$ symmetry generated by $\left(\theta_{1}\right)^{2}$. A similar computer scan for the factorizable orbifolds revealed that the list of possible symmetries given in (i)-(iii), is exhaustive.

In what follows, we show how these symmetries in the orbifold geometry affect the string couplings. For smooth, field theoretical compactifications, we know that Lorentz symmetries of the compact manifold give rise to R-symmetries in the low energy effective field theory, and that the 4D R-charges descend from the 10D Lorentz representations. This intuition has always been applied also to orbifold string compactifications, but has been worked out explicitly only in some special cases. In particular, we should check how the twisted sectors, which emerge only after the orbifold compactification and are localized in the orbifold geometry, transform under the effective R-symmetry. For example, referring to the expression for the vertex operator eq. (2.5), if we were to suppose naively that the only components of the string that transform under the 6D Lorentz transformation are $X^{i}$ and $H^{i}$, with $\partial X^{i} \rightarrow e^{2 \pi \imath v_{i}} \partial X^{i}, \partial \bar{X}^{i} \rightarrow e^{-2 \pi \imath v_{i}} \partial \bar{X}^{i}$ and $H^{i} \rightarrow H^{i}-2 \pi v^{i}$ under the Lorentz rotation $\theta_{i}$, we would infer that the picture-independent R-charges, $R^{i}=q_{s h}^{i}-\mathcal{N}_{L}^{i}+\overline{\mathcal{N}}_{L}^{i}$, should be conserved [17]. However, we would not recover in general the required trans- 


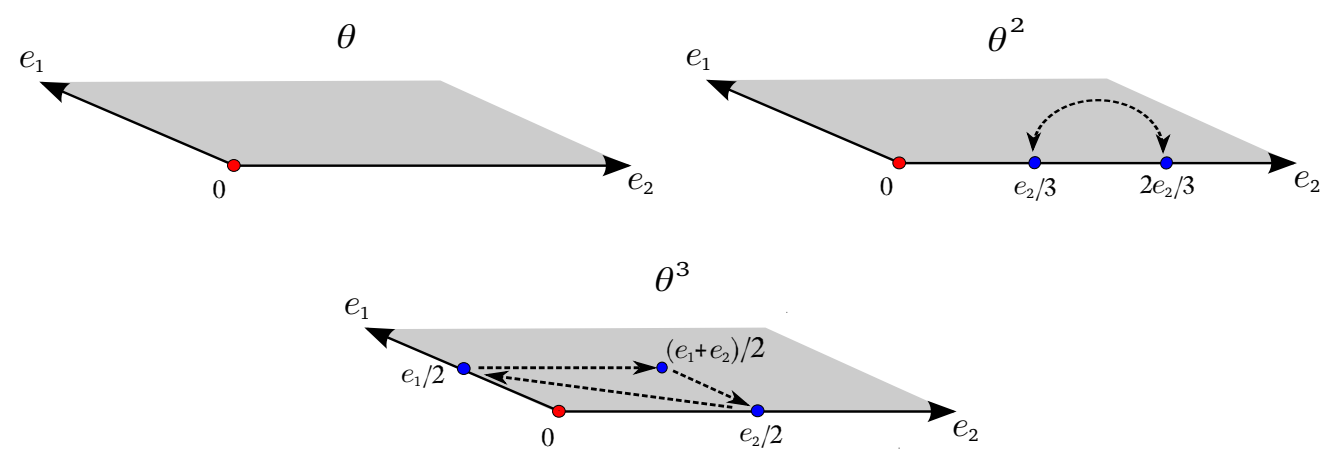

Figure 1. Fixed point structure for the various twisted sectors of the $T^{2} / \mathbb{Z}_{6}$ orbifold. Note that the fixed points in blue are related by the orbifold twist, so they are equivalent in the orbifold space.

formation under the full orbifold twist ${ }^{4} \theta_{1} \theta_{2} \theta_{3}=\theta$. To identify the R-symmetry in the effective field theory, including the R-charges carried by twisted fields, we have to look at the explicit expressions for the correlation functions.

\section{$3.2 \quad$ A $2 \mathrm{D}$ illustrative example}

It will be useful to have a simple $2 \mathrm{D}$ example in mind, before considering the general $6 \mathrm{D}$ case. As the prime orbifolds follow straightforwardly, we present a non-prime example, $T^{2} / \mathbb{Z}_{6}$ on a $G_{2}$ lattice (see figure 1 ). We focus for simplicity on the 3 -point couplings, where the explicit form of the worldsheet instanton solutions mediating the couplings is known completely.

We describe the $G_{2}$ lattice with basis vectors, $e_{1}, e_{2}$. The $\mathbb{Z}_{6}$ twist acts in the lattice basis as

$$
\theta e_{1}=-e_{1}-e_{2}, \quad \theta e_{2}=3 e_{1}+2 e_{2},
$$

and the fixed points for the first, second and third twisted sectors are:

$$
\begin{aligned}
& f^{(1)}=0, \\
& f^{(2)}=0, e_{2} / 3,2 e_{2} / 3 \\
& f^{(3)}=0, e_{2} / 2, e_{1} / 2, \frac{e_{1}+e_{2}}{2} .
\end{aligned}
$$

Thus the physical states $\left|\psi^{(k)}\right\rangle$ for the $k$-th twisted sector are (see eq. (2.4)):

$$
\begin{aligned}
& \left|\psi^{(1)}\right\rangle=|0\rangle, \\
& \left|\psi^{(2)}\right\rangle=|0\rangle,\left|e_{2} / 3\right\rangle+e^{-2 \pi \imath \gamma^{(2)}}\left|\theta\left(e_{2} / 3\right)\right\rangle, \\
& \left|\psi^{(3)}\right\rangle=|0\rangle,\left|e_{1} / 2\right\rangle+e^{-2 \pi \imath \gamma^{(3)}}\left|\theta\left(e_{1} / 2\right)\right\rangle+e^{-4 \pi \imath \gamma^{(3)}}\left|\theta^{2}\left(e_{1} / 2\right)\right\rangle,
\end{aligned}
$$

with $\gamma^{(2)} \in\{1 / 2,1\}$ and $\gamma^{(3)} \in\{1 / 3,2 / 3,1\}$.

\footnotetext{
${ }^{4}$ The same can be said for untwisted charged matter, as the orbifold twist acting in all planes together $\theta_{1} \theta_{2} \theta_{3}=\theta$, should be embedded into the gauge sector.
} 
Consider for example a coupling between physical states of type $\theta^{2} \theta^{2} \theta^{2}$, say:

$$
\left|\psi_{1}^{(2)}\right\rangle\left|\psi_{2}^{(2)}\right\rangle\left|\psi_{3}^{(2)}\right\rangle=|0\rangle\left(\left|e_{2} / 3\right\rangle+e^{-2 \pi \imath \gamma_{2}}\left|\theta\left(e_{2} / 3\right)\right\rangle\right)\left(\left|e_{2} / 3\right\rangle+e^{-2 \pi \imath \gamma_{3}}\left|\theta\left(e_{2} / 3\right)\right\rangle\right),
$$

where we suppressed the twisted sector label to lighten the notation, so that $\gamma_{\alpha}^{(2)}=\gamma_{\alpha}$. The coupling is thus given by a sum of auxiliary couplings, weighted by the $\gamma$-phases, and differing only in the positions of the fixed points. Applying the space group selection rule to each auxiliary coupling:

$$
\left(1-\theta^{k_{3}}\right) f_{3}+\theta^{k_{3}}\left(1-\theta^{k_{2}}\right) f_{2}+\theta^{k_{3}+k_{2}}\left(1-\theta^{k_{1}}\right) f_{1} \in \bigcup_{\alpha=1}^{3}\left(1-\theta^{k_{\alpha}}\right) \Lambda,
$$

one finds that the auxiliary couplings $|0\rangle\left|e_{2} / 3\right\rangle\left|e_{2} / 3\right\rangle$ and $|0\rangle\left|\theta e_{2} / 3\right\rangle\left|\theta e_{2} / 3\right\rangle$ vanish.

Now we are ready to consider the correlation functions. For a $\theta^{2} \theta^{2} \theta^{2}$ coupling only the holomorphic worldsheet instanton solutions are non-vanishing. After applying the H- and gauge-momentum conservation and Rule 5, the correlation function can be non-vanishing provided $\mathcal{N}_{L} \geq \overline{\mathcal{N}}_{L}$, and the non-trivial part of each auxiliary correlation function takes the form (see eq. (2.34)):

$$
\mathcal{F}_{\text {aux }}=\sum_{X_{c l}} e^{-S_{c l}}\left(\partial X_{c l}\right)^{\mathcal{N}_{L}-\overline{\mathcal{N}}_{L}}\left\langle\sigma_{\left(\theta^{2}, f_{1}\right)} \sigma_{\left(\theta^{2}, f_{2}\right)} \sigma_{\left(\theta^{2}, f_{3}\right)}\right\rangle,
$$

with the notation:

$$
\langle\sigma \ldots \sigma\rangle=\int \mathcal{D} X_{q u} e^{-S_{q u}} \partial X_{q u} \ldots \partial \bar{X}_{q u} \ldots \sigma \ldots \sigma,
$$

for the quantum part. The quantum part constitutes a global factor for all the auxiliary correlation functions, as it does not depend on the positions of the fixed points, but only, via the local monodromy, to which twisted sectors they belong [11, 36, 38, 39]. We are interested instead in the classical contribution, and in particular in the sum over worldsheet instanton solutions, which depends on the fixed points at which the twisted strings are localized.

The sum over classical solutions corresponds to a sum over lattice coset vectors, which for our couplings $|0\rangle\left|e_{2} / 3\right\rangle\left|\theta e_{2} / 3\right\rangle$ and $|0\rangle\left|\theta e_{2} / 3\right\rangle\left|e_{2} / 3\right\rangle$ are given, respectively, by (see eq. (2.31)):

$$
\begin{aligned}
& \nu_{1}=\left(1-\theta^{2}\right)\left(e_{2} / 3-2 e_{1}-e_{2}+\Lambda\right), \\
& \nu_{2}=\left(1-\theta^{2}\right)\left(2 e_{2} / 3-e_{1}-e_{2}+\Lambda\right) .
\end{aligned}
$$

Notice that these are related by a $\theta$-twist, $\theta \nu_{1}=\nu_{2}$, as they must be since the fixed points are related by $\theta\left\{0, e_{2} / 3, \theta e_{2} / 3\right\}=\left\{0, \theta e_{2} / 3, e_{2} / 3\right\}$. Moreover, it is easy to check that the sets of vectors $\nu_{1}$ and $\nu_{2}$ each have a $\mathbb{Z}_{3}$ rotation symmetry, generated by $\theta^{2}$, as they must since $\theta^{2}\left\{0, e_{2} / 3, \theta e_{2} / 3\right\}=\left\{0, e_{2} / 3, \theta e_{2} / 3\right\}$ and $\theta^{2}\left\{0, \theta e_{2} / 3, e_{2} / 3\right\}=\left\{0, \theta e_{2} / 3, e_{2} / 3\right\}$.

We can use these relations to combine the auxiliary correlation functions:

$$
\begin{aligned}
\mathcal{F}=e^{-2 \pi \imath \gamma_{3}} & \sum_{X_{c l}} e^{-S_{c l}}\left(\partial X_{c l}\right)^{\mathcal{N}_{L}-\overline{\mathcal{N}}_{L}}\left\langle\sigma_{\left(\theta^{2}, 0\right)} \sigma_{\left(\theta^{2}, e_{1} / 3\right)} \sigma_{\left(\theta^{2}, \theta e_{1} / 3\right)}\right\rangle \\
& +e^{-2 \pi \imath \gamma_{2}} \sum_{X_{c l}} e^{-S_{c l}}\left(\partial X_{c l}\right)^{\mathcal{N}_{L}-\overline{\mathcal{N}}_{L}}\left\langle\sigma_{\left(\theta^{2}, 0\right)} \sigma_{\left(\theta^{2}, \theta e_{1} / 3\right)} \sigma_{\left(\theta^{2}, e_{1} / 3\right)}\right\rangle,
\end{aligned}
$$


finding an overall factor:

$$
\begin{gathered}
\mathcal{F} \sim e^{-2 \pi \imath \gamma_{3}}\left((1)^{\mathcal{N}_{L}-\overline{\mathcal{N}}_{L}}+\left(\omega^{2}\right)^{\mathcal{N}_{L}-\overline{\mathcal{N}}_{L}}+\left(\omega^{4}\right)^{\mathcal{N}_{L}-\overline{\mathcal{N}}_{L}}\right) \\
+e^{-2 \pi \imath \gamma_{2}}\left((\omega)^{\mathcal{N}_{L}-\overline{\mathcal{N}}_{L}}+\left(\omega^{3}\right)^{\mathcal{N}_{L}-\overline{\mathcal{N}}_{L}}+\left(\omega^{5}\right)^{\mathcal{N}_{L}-\overline{\mathcal{N}}_{L}}\right) \\
=(\omega)^{-6 \gamma_{3}}\left(1+\omega^{\mathcal{N}_{L}-\overline{\mathcal{N}}_{L}-6 \sum_{\alpha} \gamma_{\alpha}}+\omega^{2\left(\mathcal{N}_{L}-\overline{\mathcal{N}}_{L}-6 \sum_{\alpha} \gamma_{\alpha}\right)}+\omega^{3\left(\mathcal{N}_{L}-\overline{\mathcal{N}}_{L}-6 \sum_{\alpha} \gamma_{\alpha}\right)}\right. \\
\left.+\omega^{4\left(\mathcal{N}_{L}-\overline{\mathcal{N}}_{L}-6 \sum_{\alpha} \gamma_{\alpha}\right)}+\omega^{5\left(\mathcal{N}_{L}-\overline{\mathcal{N}}_{L}-6 \sum_{\alpha} \gamma_{\alpha}\right)}\right)
\end{gathered}
$$

with $\omega=e^{2 \pi \imath / 6}$, where we recall that $\mathcal{N}_{L}$ is shorthand for $\sum_{\alpha} \mathcal{N}_{L \alpha}$, and $\gamma_{2,3}$ are shorthand for $\gamma_{2,3}^{(2)}$ so that $\gamma_{2,3} \in\{1 / 2,1\}$. From the first line of eq. (3.16), by using the geometric series, it follows that correlation functions are vanishing unless:

$$
\sum_{\alpha=1}^{3} \mathcal{N}_{L \alpha}-\overline{\mathcal{N}}_{L \alpha}=0 \bmod 3,
$$

where we have reinstated the indices in the oscillator numbers. Similarly, from the second line of eq. (3.16), we require:

$$
\sum_{\alpha=1}^{3} \mathcal{N}_{L \alpha}-\overline{\mathcal{N}}_{L \alpha}-6 \gamma_{\alpha}=0 \bmod 6,
$$

for non-vanishing correlation functions. Notice that the above conditions (3.17), (3.18) are not independent in the simple case under consideration, but we will see below $6 \mathrm{D}$ examples where the analogous conditions are independent.

Couplings of the kind $\theta \theta^{2} \theta^{3}$ can be analysed in a similar way. Consider for example the coupling:

$$
|0\rangle\left(\left|e_{2} / 3\right\rangle+e^{-2 \pi \imath \gamma_{2}}\left|\theta\left(e_{2} / 3\right)\right\rangle\right)\left(\left|e_{1} / 2\right\rangle+e^{-2 \pi \imath \gamma_{3}}\left|\theta\left(e_{1} / 2\right)\right\rangle+e^{-4 \pi \imath \gamma_{3}}\left|\theta^{2}\left(e_{1} / 2\right)\right\rangle\right) .
$$

This time, the space group selection rule allows each of the auxiliary couplings. The nontrivial part of the auxiliary correlation functions are:

$$
\mathcal{F}_{\text {aux }}=\sum_{X_{c l}} e^{-S_{c l}}\left(\partial X_{c l}\right)^{\mathcal{N}_{L}-\overline{\mathcal{N}}_{L}}\left\langle\sigma_{\left(\theta, f_{1}\right)} \sigma_{\left(\theta^{2}, f_{2}\right)} \sigma_{\left(\theta^{3}, f_{3}\right)}\right\rangle,
$$

with the sum over lattice coset vectors for $|0\rangle\left|e_{1} / 3\right\rangle\left|e_{1} / 2\right\rangle, \quad|0\rangle\left|e_{1} / 3\right\rangle\left|\theta e_{1} / 2\right\rangle$, $|0\rangle\left|e_{1} / 3\right\rangle\left|\theta^{2} e_{1} / 2\right\rangle,|0\rangle\left|\theta e_{1} / 3\right\rangle\left|e_{1} / 2\right\rangle,|0\rangle\left|\theta e_{1} / 3\right\rangle\left|\theta e_{1} / 2\right\rangle,|0\rangle\left|\theta e_{1} / 3\right\rangle\left|\theta^{2} e_{1} / 2\right\rangle$, given respectively by:

$$
\begin{aligned}
& \nu_{1}=\left(e_{1} / 3+e_{1}+2 \Lambda\right), \\
& \nu_{2}=\left(e_{1} / 3+2 \Lambda\right), \\
& \nu_{3}=\left(e_{1} / 3-e_{1}+e_{2}+2 \Lambda\right), \\
& \nu_{4}=\left(2 e_{1} / 3+e_{1}+e_{2}+2 \Lambda\right), \\
& \nu_{5}=\left(2 e_{1} / 3+e_{2}+2 \Lambda\right), \\
& \nu_{6}=\left(2 e_{1} / 3-e_{1}+e_{2}+2 \Lambda\right) .
\end{aligned}
$$


We observe that

$$
\nu_{1} \rightarrow \nu_{5} \rightarrow \nu_{3} \rightarrow \nu_{4} \rightarrow \nu_{2} \rightarrow \nu_{6}
$$

under the $\theta$ twist, as must be since the fixed points are related as:

$$
\begin{aligned}
& \left\{0, e_{1} / 3, e_{1} / 2\right\} \rightarrow\left\{0, \theta e_{1} / 3, \theta e_{1} / 2\right\} \rightarrow\left\{0, e_{1} / 3, \theta^{2} e_{1} / 2\right\} \\
& \quad \rightarrow\left\{0, \theta e_{1} / 3, e_{1} / 2\right\} \rightarrow\left\{0, e_{1} / 3, \theta e_{1} / 2\right\} \rightarrow\left\{0, \theta e_{1} / 3 \theta^{2} e_{1} / 2\right\} .
\end{aligned}
$$

Then we find the correlation function can be written with an overall factor:

$$
\begin{gathered}
\mathcal{F} \sim\left((1)^{\mathcal{N}_{L}-\overline{\mathcal{N}}_{L}}+e^{-2 \pi \imath \gamma_{3}}\left(\omega^{4}\right)^{\mathcal{N}_{L}-\overline{\mathcal{N}}_{L}}+e^{-4 \pi \imath \gamma_{3}}\left(\omega^{2}\right)^{\mathcal{N}_{L}-\overline{\mathcal{N}}_{L}}+e^{-2 \pi \imath \gamma_{2}}\left(\omega^{3}\right)^{\mathcal{N}_{L}-\overline{\mathcal{N}}_{L}}\right. \\
\left.+e^{-2 \pi \imath \gamma_{2}-2 \pi \imath \gamma_{3}}(\omega)^{\mathcal{N}_{L}-\overline{\mathcal{N}}_{L}}+e^{-2 \pi \imath \gamma_{2}-4 \pi \imath \gamma_{3}}\left(\omega^{5}\right)^{\mathcal{N}_{L}-\overline{\mathcal{N}}_{L}}\right) \\
\sim\left(1+\omega^{\mathcal{N}_{L}-\overline{\mathcal{N}}_{L}-6 \sum_{\alpha} \gamma_{\alpha}}+\omega^{2\left(\mathcal{N}_{L}-\overline{\mathcal{N}}_{L}-6 \sum_{\alpha} \gamma_{\alpha}\right)}+\omega^{3\left(\mathcal{N}_{L}-\overline{\mathcal{N}}_{L}-6 \sum_{\alpha} \gamma_{\alpha}\right)}\right. \\
\left.+\omega^{4\left(\mathcal{N}_{L}-\overline{\mathcal{N}}_{L}-6 \sum_{\alpha} \gamma_{\alpha}\right)}+\omega^{5\left(\mathcal{N}_{L}-\overline{\mathcal{N}}_{L}-6 \sum_{\alpha} \gamma_{\alpha}\right)}\right),
\end{gathered}
$$

and hence the correlation functions are vanishing unless:

$$
\sum_{\alpha=1}^{3} \mathcal{N}_{L \alpha}-\overline{\mathcal{N}}_{L \alpha}-6 \gamma_{\alpha}=0 \bmod 6 .
$$

Finally, we can make a similar analysis for the $\theta \theta \theta^{4}$ couplings, with the result:

$$
\begin{aligned}
& \sum_{\alpha=1}^{3} \mathcal{N}_{L \alpha}-\overline{\mathcal{N}}_{L \alpha}=0 \bmod 3, \\
& \sum_{\alpha=1}^{3} \mathcal{N}_{L \alpha}-\overline{\mathcal{N}}_{L \alpha}-6 \gamma_{\alpha}=0 \bmod 6,
\end{aligned}
$$

where again the two conditions are not independent.

In summary, we find that the symmetries relating the orbifold fixed points lead to symmetries among the worldsheet instanton solutions, which imply that all couplings are vanishing unless

$$
\sum_{\alpha=1}^{3} \mathcal{N}_{L \alpha}-\overline{\mathcal{N}}_{L \alpha}-6 \gamma_{\alpha}=0 \bmod 6 .
$$

If we combine this selection rule with the H-momentum conservation condition ${ }^{5}$ (2.14), we can write it as an R-charge conservation law:

$$
\sum_{\alpha} R_{\alpha}=1 \bmod 6 \quad \text { with } \quad R_{\alpha}=q_{s h \alpha}-\mathcal{N}_{L \alpha}+\overline{\mathcal{N}}_{L \alpha}+6 \gamma_{\alpha} .
$$

Importantly and not to be missed, the $\gamma$-phases contribute non-trivially to the R-charges.

Notice that in our simple 2D example, the orbifold geometrical symmetry under discussion is nothing more than the orbifold twist symmetry itself, and yet we are led to

\footnotetext{
${ }^{5} \mathrm{~A}$ priori, for the 3-point couplings, we must still impose H-momentum conservation as an independent selection rule, see comment below eq. (2.14), and a further comment in what follows.
} 
conditions that are in general independent of the orbifold projection condition, the 2D version of eq. (2.9), which reads:

$$
\sum_{\alpha} \mathcal{N}_{L \alpha}-\overline{\mathcal{N}}_{L \alpha}+6 \gamma_{\alpha}=0 \bmod 6
$$

after application of gauge and H-momentum conservation and the space group selection rule. ${ }^{6}$ The orbifold projection condition, eq. (3.30), is satisfied automatically for any combination of physical states. In contrast, the R-charge conservation law, eqs. (3.28) or (3.29), follows from the structure of the correlation function, and is due to the relations between fixed points or worldsheet instantons. For prime orbifolds, where there is no need to build the physical states via the $\gamma$-phase, the R-charge conservation law is identical to the orbifold twist invariance condition in $2 \mathrm{D}$. They also happen to be identical for the $\mathbb{Z}_{4}$ case, since there we always have $\gamma \in\{0,1 / 2\}$, so that $\gamma \simeq-\gamma$. However, they are independent in general.

\subsection{The general $6 \mathrm{D}$ case}

With our simple 2D example in mind, let us now consider the general $6 \mathrm{D}$ case, where we can have additional symmetries beyond the point group, as enumerated in subsection 3.1.

A general L-point coupling between physical states,

$$
\left|\psi_{\alpha}\right\rangle=\sum_{r=0}^{l_{\alpha}-1} e^{-2 \pi \imath r \gamma_{\alpha}}\left|\theta^{r} f_{\alpha}\right\rangle
$$

can be written as a sum of auxiliary couplings, weighted by the $\gamma$-phases:

$$
\left\langle\psi_{1} \ldots \psi_{\mathrm{L}}\right\rangle=\sum_{r_{1}=0}^{l_{1}-1} \ldots \sum_{r_{\mathrm{L}}=0}^{l_{\mathrm{L}}-1} e^{-2 \pi \imath\left(r_{1} \gamma_{1}+\cdots+r_{\mathrm{L}} \gamma_{\mathrm{L}}\right)}\left\langle\theta^{r_{1}} f_{1} \ldots \theta^{r_{\mathrm{L}}} f_{\mathrm{L}}\right\rangle,
$$

where we recall that $l_{\alpha}$ is the smallest integer such that $\theta^{l_{\alpha}} f_{\alpha}=f_{\alpha}+\lambda$, and $\gamma_{\alpha}=p_{\alpha} / l_{\alpha}$ for some integers $p_{\alpha}$ in the range $0,1, \ldots, l_{\alpha}-1$.

In order to avoid being distracted by clutter, let us assume to begin with that holomorphic instantons are allowed in all planes. We will see by the end of the section that the general case follows straightforwardly. As we discussed in section 2.3 and eq. (2.34), the non-trivial part of the correlation function, which depends on oscillators and twist fields, takes the form:

$$
\begin{gathered}
\mathcal{F} \sim \sum_{r_{1}=0}^{l_{1}-1} \cdots \sum_{r_{\mathrm{L}}=0}^{l_{\mathrm{L}}-1} e^{-2 \pi \imath\left(r_{1} \gamma_{1}+\cdots+r_{\mathrm{L}} \gamma_{\mathrm{L}}\right)} \prod_{i=1}^{3} \sum_{X_{c l}^{i}} e^{-S_{c l}^{i}\left(\partial X_{c l}^{i}\right)} \\
\times\left\langle\sigma_{\left(k_{1}, \theta^{r} 1 f_{1}\right)} \ldots \sigma_{\left(k_{\mathrm{L}}, \theta^{r} \mathrm{~L} f_{\mathrm{L}}\right)}\right\rangle,
\end{gathered}
$$

when holomorphic instantons are allowed, where again note that the classical solutions depend on the fixed points $\left\{\theta^{r_{1}} f_{1}, \ldots, \theta^{r_{\mathrm{L}}} f_{\mathrm{L}}\right\}$ in each auxiliary correlation function. We may now use this expression, and the relations among the fixed points and corresponding classical solutions, to derive selection rules.

\footnotetext{
${ }^{6}$ The sum of the vacuum phases is always trivial, as can be shown via modular invariance and the space group selection rule [19].
} 


\subsubsection{Factorizable orbifolds}

The orbifolds $T^{6} / \mathbb{Z}_{3}, T^{6} / \mathbb{Z}_{4}, T^{6} / \mathbb{Z}_{6-I}$ and $T^{6} / \mathbb{Z}_{6-I I}$ may be constructed on factorizable lattices. For these factorizable orbifolds, we can rewrite eq. (3.33) as:

$$
\begin{gathered}
\mathcal{F} \sim \sum_{r_{1}=0}^{l_{1}-1} \cdots \sum_{r_{\mathrm{L}}=0}^{l_{\mathrm{L}}-1} e^{-2 \pi \imath\left(r_{1} \gamma_{1}+\cdots+r_{\mathrm{L}} \gamma_{\mathrm{L}}\right)} \prod_{i=1}^{3} \sum_{X_{c l}^{i}} e^{-S_{c l}^{i}\left(\partial X_{c l}^{i}\right)\left(\mathcal{N}_{L}^{i}-\overline{\mathcal{N}}_{L}^{i}-\overline{\mathcal{N}}_{R}^{i}\right)} f\left(\left|\partial X_{c l}^{i}\right|^{2}, \partial X_{c l}^{i} \partial \bar{X}_{c l}^{i}\right) \\
\times\left\langle\sigma_{\left(k_{1}, \theta_{i}^{i} g_{1 i}\right)}^{i} \cdots \sigma_{\left(k_{\mathrm{L}}, \theta_{i}^{\left.r_{\mathrm{L}} g_{\mathrm{L} i}\right)}\right.}^{i}\right\rangle
\end{gathered}
$$

Now, let us suppose that some planes, $j$, have prime ordered twists. Then all the fixed points are $\theta$-invariant in $j$, that is, $\theta_{j} g_{\alpha j}=g_{\alpha j}$ for all $\alpha$. It follows that the correlation function factorizes in prime planes:

$$
\begin{aligned}
& \mathcal{F} \sim \prod_{j} \sum_{X_{c l}^{j}} e^{-S_{c l}^{j}}\left(\partial X_{c l}^{j}\right)^{\left(\mathcal{N}_{L}^{j}-\overline{\mathcal{N}}_{L}^{j}-\overline{\mathcal{N}}_{R}^{j}\right)} f\left(\left|\partial X_{c l}^{j}\right|^{2}, \partial X_{c l}^{j} \partial \bar{X}_{c l}^{j}\right)\left\langle\sigma_{\left(k_{1}, g_{1 j}\right)}^{j} \ldots \sigma_{\left(k_{\mathrm{L}}, g_{\mathrm{L} j}\right)}^{j}\right\rangle
\end{aligned}
$$

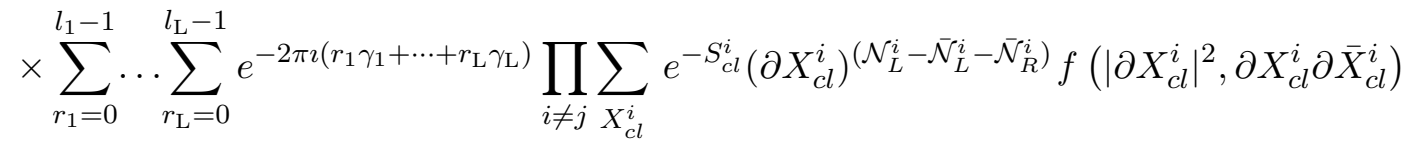

$$
\begin{aligned}
& \times\left\langle\sigma_{\left(k_{1}, \theta_{i}^{\left.r_{1} g_{1 i}\right)}\right.}^{i} \ldots \sigma_{\left(k_{\mathrm{L}}, \theta_{i}^{r_{\mathrm{L}}} g_{\mathrm{L} i}\right)}^{i}\right\rangle .
\end{aligned}
$$

Moreover, from the observations at the end of section 2.3, it can be seen that the classical solutions $\left\{\partial X_{c l}^{j}\right\}$ to be summed over are related by $\theta_{j}$ twists:

$$
\theta_{j}: \quad \partial X_{c l 1}^{j} \rightarrow \omega_{j} \partial X_{c l 1}^{j}=\partial X_{c l 2}^{j}, \quad \text { with } \omega_{j}=e^{2 \pi \imath / \mathrm{N}^{j}},
$$

where $\mathrm{N}^{j}$ is the order of the twist $\theta_{j}$. The sum over classical solutions thus leads to an overall factor:

$$
\mathcal{F} \sim\left((1)^{\left(\mathcal{N}_{L}^{j}-\overline{\mathcal{N}}_{L}^{j}-\overline{\mathcal{N}}_{R}^{j}\right)}+\left(\omega_{j}\right)^{\left(\mathcal{N}_{L}^{j}-\overline{\mathcal{N}}_{L}^{j}-\overline{\mathcal{N}}_{R}^{j}\right)}+\cdots+\left(\omega_{j}^{\left(\mathrm{N}^{j}-1\right)}\right)^{\left(\mathcal{N}_{L}^{j}-\overline{\mathcal{N}}_{L}^{j}-\overline{\mathcal{N}}_{R}^{j}\right)}\right),
$$

which implies a selection rule for the prime planes:

$$
\sum_{\alpha} \mathcal{N}_{L \alpha}^{j}-\overline{\mathcal{N}}_{L \alpha}^{j}-\overline{\mathcal{N}}_{R \alpha}^{j}=0 \bmod \mathrm{N}^{j}
$$

Subsequently, the non-vanishing correlation functions are invariant under the orbifold twist acting independently in the prime planes, which corresponds to a Lorentz symmetry in the orbifold geometry, as we saw in subsection 3.1. This is the familiar $\mathbb{Z}_{\mathrm{N}^{j}}$ twist invariance condition for prime planes, and combining with H-momentum conservation (2.14), it leads to the R-charge conservation law

$$
\sum_{\alpha} R_{\alpha}^{j}=1 \bmod \mathrm{N}^{j}, \quad \text { with } R_{\alpha}^{j}=q_{s h \alpha}^{j}-\mathcal{N}_{L \alpha}^{j}+\overline{\mathcal{N}}_{L \alpha}^{j} .
$$

To make further progress for the non-prime planes, consider a general coupling between some number $\mathrm{L}_{1}$ of twisted sectors associated with $l_{1}$ up to $\mathrm{L}_{\mathrm{L}}$ twisted sectors with $l_{\mathrm{L}}$, and take $\mathrm{K}$ the lowest common mutiple of $l_{1}, \ldots, l_{\mathrm{L}}$, so $\theta^{\mathrm{K}} f_{\alpha}=f_{\alpha}+\lambda$ for all 
the associated fixed points $f_{\alpha}$. The number of auxiliary couplings is $l_{1}^{\mathrm{L}_{1}} \times \cdots \times l_{\mathrm{L}}^{\mathrm{L}_{\mathrm{L}}}$, and these can be divided into $\left(l_{1}^{\mathrm{L}_{1}} \times \cdots \times l_{\mathrm{L}}^{\mathrm{L}_{\mathrm{L}}}\right) / \mathrm{K}$ sets, each with $\mathrm{K}$ elements whose fixed points are related by powers of $\theta$ up to $\mathrm{K}-1$. For example, for a $\theta^{2} \theta^{2} \theta^{2}$ coupling in a $\mathbb{Z}_{6}$ orbifold, we have $\left\{\left\{f_{1}, f_{2}, f_{3}\right\},\left\{\theta f_{1}, \theta f_{2}, \theta f_{3}\right\}\right\},\left\{\left\{f_{1}, f_{2}, \theta f_{3}\right\},\left\{\theta f_{1}, \theta f_{2}, f_{3}\right\}\right\}$, $\left\{\left\{f_{1}, \theta f_{2}, f_{3}\right\},\left\{\theta f_{1}, f_{2}, \theta f_{3}\right\}\right\}$ and $\left\{\left\{\theta f_{1}, f_{2}, f_{3}\right\},\left\{f_{1} \theta f_{2}, \theta f_{3}\right\}\right\}$. If one element of a set is allowed by the space group selection rule, then all its elements are allowed. The classical solutions corresponding to the auxiliary correlation functions will be similarly related, so for example, for $\left\{f_{1}, f_{2}, f_{3}\right\}$ and $\left\{\theta f_{1}, \theta f_{2}, \theta f_{3}\right\}$ we have, respectively,

$$
\partial X_{c l}=\left(\partial X_{c l}^{1}, \partial X_{c l}^{2}, \partial X_{c l}^{3}\right) \quad \text { and } \quad \theta \partial X_{c l}=\left(e^{2 \pi \imath v_{1}} \partial X_{c l}^{1}, e^{2 \pi \imath v_{2}} \partial X_{c l}^{2}, e^{2 \pi \imath v_{3}} \partial X_{c l}^{3}\right)
$$

Recall from subsection 3.1 that we cannot factorize the $\theta$-twist plane by plane for nonprime planes.

Moreover, since all the fixed points are invariant under $\theta^{\mathrm{K}}$, it follows that the classical solutions corresponding to each auxiliary correlation function are related by powers of $\theta^{\mathrm{K}}$, up to ${ }^{7}\left(\theta^{\mathrm{K}}\right)^{\frac{\mathrm{N}}{\mathrm{K}}-1}$. For the $\theta^{2} \theta^{2} \theta^{2}$ example, this corresponds to an additional $\mathbb{Z}_{3}$ symmetry, generated by $\theta^{2}$. We can write this $\theta^{\mathrm{K}}$ symmetry among the classical solutions plane by plane (cf. eq. (3.5)), and thus the sum over classical solutions leads to an overall factor:

$$
\mathcal{F}_{\text {aux }} \sim \prod_{i \neq j}\left((1)^{\left(\mathcal{N}_{L}^{i}-\overline{\mathcal{N}}_{L}^{i}-\overline{\mathcal{N}}_{R}^{i}\right)}+\left(\omega_{i}\right)^{\left(\mathcal{N}_{L}^{i}-\overline{\mathcal{N}}_{L}^{i}-\overline{\mathcal{N}}_{R}^{i}\right)}+\cdots+\left(\omega_{i}^{\left(\frac{\mathrm{N}^{i}}{\mathrm{~K}}-1\right)}\right)^{\left(\mathcal{N}_{L}^{i}-\overline{\mathcal{N}}_{L}^{i}-\overline{\mathcal{N}}_{R}^{i}\right)}\right)
$$

with $\omega_{i}=e^{2 \pi i \frac{\mathrm{K}}{\mathrm{N}^{i}}}$. Therefore, in each of the planes with non-prime twist $\mathrm{N}^{i}$, we have the condition:

$$
\sum_{\alpha} \mathcal{N}_{L \alpha}^{i}-\overline{\mathcal{N}}_{L \alpha}^{i}-\overline{\mathcal{N}}_{R \alpha}^{i}=0 \bmod \frac{\mathrm{N}^{i}}{\mathrm{~K}}
$$

Next let us combine all the auxiliary correlation functions. We need the relations amongst the classical solutions, but also amongst the $\gamma$-phase weightings. Consider for example an auxiliary coupling, with fixed points $\left\{\theta^{r_{1}} f_{1}, \ldots, \theta^{r_{\mathrm{L}}} f_{\mathrm{L}}\right\}$ that are related to the $\left\{f_{1}, f_{2}, \ldots, f_{\mathrm{L}}\right\}$ by some twist $\theta^{s}$. We have seen that the classical solutions mediating the first coupling are related to the second by a rotation $\theta^{s}$, and that there are $\mathrm{K}$ such couplings, with $s=0, \ldots, \mathrm{K}-1$. Now, the integer $s$ is related to the integers $r_{\alpha}$ as $\theta^{r_{\alpha}} f_{\alpha}=\theta^{s} f_{\alpha}+\lambda$ for all $\alpha$, that is $s-r_{\alpha}=m l_{\alpha}$ with integer $m$. Thus, the $\gamma$-phase weighting factor can be written as

$$
e^{-2 \pi \imath\left(r_{1} \gamma_{1}+\cdots+r_{\mathrm{L}} \gamma_{\mathrm{L}}\right)}=e^{-2 \pi \imath s \sum_{\alpha} \gamma_{\alpha}},
$$

where we have used that $\gamma_{\alpha}$ is an integer multiple of $1 / l_{\alpha}$. Moreover, as we discussed above, if the fixed points $\left\{f_{1}, f_{2}, \ldots, f_{\mathrm{L}}\right\}$ are all invariant under $\theta^{\mathrm{K}}$, then the classical solutions in each auxiliary coupling have a $\theta^{\mathrm{K}}$ symmetry. These do not come with $\gamma$-phases weighting their relative contributions, but we can use $\mathrm{K}=m l_{\alpha}$ with integer $m$ for all $\alpha$, to insert factors of $1=e^{-2 \pi \imath \mathrm{K} \sum_{\alpha} \gamma_{\alpha}}$.

Let us now apply all these relations to simplify the non-prime part of the full correlation function (3.35). We may put together the classical solutions from each set of auxiliary

\footnotetext{
${ }^{7}$ Notice that since all the $l_{\alpha}$ divide $\mathrm{N}$, by prime factorization so does their lowest common multiple K.
} 
correlation functions that are related by $1, \theta, \ldots, \theta^{\mathrm{K}-1}$, and moreover from within each auxiliary correlation function itself, which are related by $1, \theta^{\mathrm{K}}, \ldots,\left(\theta^{\mathrm{K}}\right)^{\frac{\mathrm{N}}{\mathrm{K}}-1}$. Combined, the classical solutions are all related by $1, \theta, \ldots, \theta^{\mathrm{N}-1}$. Taking care of the $\gamma$-phase weightings, one thus finds that the correlation function can be written with an overall factor:

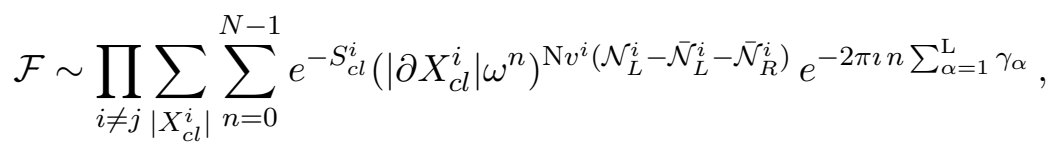

where $\omega=e^{2 \pi \imath / \mathrm{N}}$, and we have split the sum over classical solutions into a sum over phases and lengths. From here one concludes immediately that correlation functions vanish unless:

$$
\sum_{\alpha}\left(\sum_{i \neq j} \mathrm{~N} v^{i}\left(\mathcal{N}_{L \alpha}^{i}-\overline{\mathcal{N}}_{L \alpha}^{i}-\overline{\mathcal{N}}_{R \alpha}^{i}\right)-\mathrm{N} \gamma_{\alpha}\right)=0 \bmod \mathrm{N}
$$

This rule holds for all couplings, and putting together with H-momentum conservation (2.14), it can be expressed as an R-charge conservation law:

$$
\sum_{\alpha} R_{\alpha}=\left(\sum_{i \neq j} \mathrm{~N} v^{i}\right) \bmod \mathrm{N} \quad \text { with } R_{\alpha}=\sum_{i \neq j} \mathrm{~N} v^{i}\left(q_{s h \alpha}^{i}-\mathcal{N}_{L \alpha}^{i}+\overline{\mathcal{N}}_{L \alpha}^{i}\right)+\mathrm{N} \gamma_{\alpha},
$$

Observe that the correct R-charge for non-prime planes is summed over the non-prime planes, and has a non-trivial contribution from the $\gamma$-phase.

Notice that with at most one non-prime plane, the condition (3.42) follows automatically after imposing the R-charge conservation law or (3.45). However, when there are two non-prime planes, the condition (3.42) becomes an independent selection rule, which we may call Rule 6 or the coset vector selection rule. Since this condition depends on the relative properties of the twisted sectors involved in the couplings, namely the lowest common multiple of the twisted sectors (or their conjugates), it cannot be interpreted as a conventional symmetry like R-symmetry in the $4 \mathrm{D}$ theory. It is however a stringy selection rule, coming from the symmetries in the fixed points or worldsheet instantons.

To complete our analysis, so far in deriving the rules we assumed for simplicity that holomorphic instantons were allowed in every plane. In general, holomorphic instantons may be forbidden in some (or all) planes, in which case the correlation functions also include factors of the form eq. (2.35). The classical solutions to be summed over in the correlation functions are related by the $\theta$ twist as follows. For $\left\{f_{1}, f_{2}, f_{3}\right\}$ and $\left\{\theta f_{1}, \theta f_{2}, \theta f_{3}\right\}$ we have, respectively,

$$
\begin{aligned}
& \partial X_{c l}=\left(\partial X_{c l}^{1}, \partial X_{c l}^{2}, \partial X_{c l}^{3}\right) \\
& \partial \bar{X}_{c l}=\left(\partial \bar{X}_{c l}^{1}, \partial \bar{X}_{c l}^{2}, \partial \bar{X}_{c l}^{3}\right)
\end{aligned}
$$

and

$$
\begin{aligned}
& \theta \partial X_{c l}=\left(e^{2 \pi \imath v_{1}} \partial X_{c l}^{1}, e^{2 \pi \imath v_{2}} \partial X_{c l}^{2}, e^{2 \pi \imath v_{3}} \partial X_{c l}^{3}\right), \\
& \theta \partial \bar{X}_{c l}=\left(e^{-2 \pi \imath v_{1}} \partial \bar{X}_{c l}^{1}, e^{-2 \pi \imath v_{2}} \partial \bar{X}_{c l}^{2}, e^{-2 \pi \imath v_{3}} \partial \bar{X}_{c l}^{3}\right) .
\end{aligned}
$$


From here, it follows that the selection rules in the general case take the same form found above, eqs. (3.39), (3.42), (3.46).

Finally, we have already discussed how H-momentum conservation is not a selection rule for higher order couplings. Following an analogous proof to that found in [13], it can be shown that even for three-point couplings, it is sufficient to impose R-charge conservation and the point group selection rule, to ensure that $\mathrm{H}$-momentum conservation is automatically satisfied.

We are now ready to put all we have learned together, to write down the instanton rules for some representative examples. We refer to $[35,36]$ for details on the torus lattices, twist actions and fixed points.

$T^{6} / \mathbb{Z}_{3}$ on $\mathrm{SU}(3) \times \mathrm{SU}(3) \times \mathrm{SU}(3)$ lattice. Since this is a prime orbifold, there is no need to build the physical states via the $\gamma$-phases. The correlation function factorizes into all three complex planes, and the R-charge conservation laws are as expected [14-16]:

$$
\sum_{\alpha} R_{\alpha}^{i}=1 \bmod 3 \quad \text { with } R_{\alpha}^{i}=q_{s h \alpha}^{i}-\mathcal{N}_{L \alpha}^{i}+\overline{\mathcal{N}}_{L \alpha}^{i}, \quad i=1,2,3 .
$$

Notice that, for the prime factorizable orbifold, we have an R-charge condition for each plane. The sum of the three conditions is equivalent to the orbifold projection condition, which is automatically satisfied for all possible combinations of states.

$T^{6} / \mathbb{Z}_{4}$ on $\mathrm{SO}(4) \times \mathrm{SO}(4) \times \mathrm{SO}(4)$ lattice. Only the third plane is prime, allowing us to factorize the correlation function there. The R-charge conservation law is:

$$
\begin{aligned}
& \sum_{\alpha} R_{\alpha}=2 \bmod 4, \quad \text { with } R_{\alpha}=\sum_{i=1}^{2}\left(q_{s h \alpha}^{i}-\mathcal{N}_{L \alpha}^{i}+\overline{\mathcal{N}}_{L \alpha}^{i}\right)+4 \gamma_{\alpha} \\
& \sum_{\alpha} R_{\alpha}^{3}=1 \bmod 2, \quad \text { with } R_{\alpha}^{3}=q_{s h \alpha}^{3}-\mathcal{N}_{L \alpha}^{3}+\overline{\mathcal{N}}_{L \alpha}^{3} \quad \text { (not independent) },
\end{aligned}
$$

and moreover

$$
\begin{aligned}
& \sum_{\alpha} R_{\alpha}^{1}=1 \bmod 2, \quad \text { with } R_{\alpha}^{1}=q_{s h \alpha}^{1}-\mathcal{N}_{L \alpha}^{1}+\overline{\mathcal{N}}_{L \alpha}^{1} \\
& \sum_{\alpha} R_{\alpha}^{2}=1 \bmod 2, \quad \text { with } R_{\alpha}^{2}=q_{s h \alpha}^{2}-\mathcal{N}_{L \alpha}^{2}+\overline{\mathcal{N}}_{L \alpha}^{2} \quad \text { (not independent) }
\end{aligned}
$$

since all states are twisted under either $\theta$ or $\theta^{2}$.

$T^{6} / \mathbb{Z}_{6-I}$ on $G_{2} \times G_{2} \times \operatorname{SO}(4)$ lattice. Again, only the third plane is prime, and so the correlation function in general only factorizes there. The R-charge conservation laws are:

$$
\begin{aligned}
& \sum_{\alpha} R_{\alpha}^{3}=1 \bmod 3, \quad \text { with } R_{\alpha}^{3}=q_{s h \alpha}^{3}-\mathcal{N}_{L \alpha}^{3}+\overline{\mathcal{N}}_{L \alpha}^{3} \\
& \sum_{\alpha} R_{\alpha}=2 \bmod 6, \quad \text { with } R_{\alpha}=\sum_{i=1}^{2}\left(q_{s h \alpha}^{i}-\mathcal{N}_{L \alpha}^{i}+\overline{\mathcal{N}}_{L \alpha}^{i}\right)+6 \gamma_{\alpha} .
\end{aligned}
$$


Moreover, for couplings involving only $\theta, \theta^{2}$ (and untwisted) sectors, we have the additional stringy Rule 6:

$$
\begin{aligned}
& \sum_{\alpha} \mathcal{N}_{L \alpha}^{1}-\overline{\mathcal{N}}_{L \alpha}^{1}-\overline{\mathcal{N}}_{R \alpha}^{1}=0 \bmod 3, \\
& \sum_{\alpha} \mathcal{N}_{L \alpha}^{2}-\overline{\mathcal{N}}_{L \alpha}^{2}-\overline{\mathcal{N}}_{R \alpha}^{2}=0 \bmod 3 \quad \text { (not independent), }
\end{aligned}
$$

and for couplings involving only $\theta, \theta^{3}$ (and untwisted) sectors, we have:

$$
\begin{aligned}
& \sum_{\alpha} \mathcal{N}_{L \alpha}^{1}-\overline{\mathcal{N}}_{L \alpha}^{1}-\overline{\mathcal{N}}_{R \alpha}^{1}=0 \bmod 2 \\
& \sum_{\alpha} \mathcal{N}_{L \alpha}^{2}-\overline{\mathcal{N}}_{L \alpha}^{2}-\overline{\mathcal{N}}_{R \alpha}^{2}=0 \bmod 2 \quad \text { (not independent). }
\end{aligned}
$$

As these rules are coupling dependent, they cannot be interpreted as a charge conservation law and symmetry in the low energy effective field theory.

$T^{6} / \mathbb{Z}_{6-I I}$ on $G_{2} \times \mathrm{SU}(3) \times \mathrm{SO}(4)$ lattice. This orbifold has only one non-prime plane, and hence the correlation function factorizes fully as in the $\mathbb{Z}_{3}$ case. There are three independent R-charge conservation laws:

$$
\begin{array}{ll}
\sum_{\alpha} R_{\alpha}^{1}=1 \bmod 6 & \text { with } R_{\alpha}^{1}=q_{s h \alpha}^{1}-\mathcal{N}_{L \alpha}^{1}+\overline{\mathcal{N}}_{L \alpha}^{1}+6 \gamma_{\alpha}, \\
\sum_{\alpha} R_{\alpha}^{2}=1 \bmod 3 & \text { with } R_{\alpha}^{2}=q_{s h \alpha}^{2}-\mathcal{N}_{L \alpha}^{2}+\overline{\mathcal{N}}_{L \alpha}^{2} \\
\sum_{\alpha} R_{\alpha}^{3}=1 \bmod 2 & \text { with } R_{\alpha}^{3}=q_{s h \alpha}^{3}-\mathcal{N}_{L \alpha}^{3}+\overline{\mathcal{N}}_{L \alpha}^{3} .
\end{array}
$$

The $\gamma$-phase contributes non-trivially to the R-charges of the non-prime plane. The conditions are independent of the orbifold projection condition.

\subsubsection{Non-factorizable orbifolds}

As we observed in subsection 3.1, for the non-factorizable orbifolds the twist does not act plane by plane. However, by definition, the fixed points are invariant under, or related by, the twist acting in all the planes at the same time. Moreover, in some cases, we found additional symmetries that leave the fixed points invariant.

Let us first consider the consequences of the orbifold twist symmetry in both the prime and non-prime cases. For the prime cases, this leads only to the orbifold projection condition, which is satisfied by all possible combinations of physical states. Thus we have no R-charge conservation law or Rule 6 for the non-factorizable, prime orbifolds. However, for the non-prime orbifolds, the orbifold twist does give rise to additional selection rules. The derivation is exactly analogous to the factorizable case, but now, as the twist does not act plane by plane, all the planes must be taken together.

In detail, consider a coupling between twisted sectors that are all fixed under $\theta^{\mathrm{K}}$. The set of classical instanton solutions $\partial X_{c l}$ for each auxiliary function enjoys the discrete symmetry generated by $\theta^{\mathrm{K}}$, with

$$
\theta^{\mathrm{K}} \partial X_{c l}=\left(e^{2 \pi \imath \mathrm{K} v^{1}} \partial X_{c l}^{1}, e^{2 \pi \imath \mathrm{K} v^{2}} \partial X_{c l}^{2}, e^{2 \pi \imath \mathrm{K} v^{3}} \partial X_{c l}^{3}\right),
$$


but now it cannot be written plane by plane as in eq. (3.41). Instead, each auxiliary correlation function has an overall factor:

$$
\begin{aligned}
\mathcal{F}_{\text {aux }} \sim\left((1)^{\sum_{i=1}^{3} \mathrm{~N} v^{i}\left(\mathcal{N}_{L}^{i}-\overline{\mathcal{N}}_{L}^{i}-\overline{\mathcal{N}}_{R}^{i}\right)}+(\omega)^{\sum_{i=1}^{3} \mathrm{~N} v^{i}\left(\mathcal{N}_{L}^{i}-\overline{\mathcal{N}}_{L}^{i}-\overline{\mathcal{N}}_{R}^{i}\right)}+\ldots\right. \\
\left.\ldots+\left(\omega^{\left(\frac{\mathrm{N}}{\mathrm{K}}-1\right)}\right)^{\sum_{i=1}^{3} \mathrm{~N} v^{i}\left(\mathcal{N}_{L}^{i}-\overline{\mathcal{N}}_{L}^{i}-\overline{\mathcal{N}}_{R}^{i}\right)}\right), \\
\cdots
\end{aligned}
$$

with $\omega=e^{2 \pi \imath \frac{\mathrm{K}}{\mathrm{N}}}$. From this expression the condition:

$$
\sum_{i=1}^{3} \mathrm{~N} v^{i}\left(\mathcal{N}_{L}^{i}-\overline{\mathcal{N}}_{L}^{i}-\overline{\mathcal{N}}_{R}^{i}\right)=0 \bmod \frac{\mathrm{N}}{\mathrm{K}},
$$

immediately follows.

Now, in a similar way, by putting together the classical solutions from all the auxiliary correlation functions, we can derive a $Q$-charge conservation condition:

$$
\sum_{\alpha} Q_{\alpha}=0 \bmod \mathrm{N}, \quad \text { with } \quad Q_{\alpha}=\sum_{i=1}^{3} \mathrm{~N} v^{i}\left(q_{s h \alpha}^{i}-\mathcal{N}_{L \alpha}^{i}+\overline{\mathcal{N}}_{L \alpha}^{i}\right)+\mathrm{N} \gamma_{\alpha} .
$$

Using the twist vectors given in table 1 , and the relation between $\mathrm{H}$-momentum in the spinor and vector representations (2.7), one sees that the charges $Q_{\alpha}$ are the same for bosonic and fermionic superpartners. Hence, the charges $Q_{\alpha}$ do not correspond to Rcharges. However, their conservation does constitute a non-trivial coupling selection rule, independent of the orbifold projection condition, and corresponding to a symmetry in the low energy effective field theory.

Finally, one can use the relation $\gamma_{\alpha} \mathrm{K}=$ integer to show that the condition (3.65) is always satisfied once the charge conservation law (3.66) is imposed, so Rule 6 is trivial for the non-factorizable orbifolds.

Let us then give some examples, including some orbifolds whose underlying lattice and twist action is partially factorizable.

$T^{6} / \mathbb{Z}_{\mathbf{7}}$ on $\mathrm{SU}(\mathbf{7})$ lattice. This orbifold is both non-factorizable and prime, and hence we expect no Q- or R-charge selection rule and no Rule 6.

$T^{6} / \mathbb{Z}_{6-I I}$ on $\mathrm{SU}(6) \times \mathrm{SU}(2)$ lattice. This orbifold is non-factorizable, but it is nonprime. Hence, we derive an Q-charge conservation rule:

$$
\sum_{\alpha} Q_{\alpha}=0 \bmod 6, \quad \text { with } Q_{\alpha}=\sum_{i=1}^{3} 6 v^{i}\left(q_{s h \alpha}^{i}-\mathcal{N}_{L \alpha}^{i}+\overline{\mathcal{N}}_{L \alpha}^{i}\right)+6 \gamma_{\alpha},
$$

which is different to the orbifold projection condition.

$T^{6} / \mathbb{Z}_{8-I I}$ on $\mathrm{SO}(8) \times \mathbf{S O}(4)$ lattice. This orbifold is partially factorizable, as both the underlying lattice and orbifold twist can be factorized on the third complex plane, where the twist is prime order 2 . Thus we obtain the R-charge conservation rules:

$$
\begin{aligned}
& \sum_{\alpha} R_{\alpha}=4 \bmod 8, \quad \text { with } R_{\alpha}=\sum_{i=1}^{2} 8 v^{i}\left(q_{s h \alpha}^{i}-\mathcal{N}_{L \alpha}^{i}+\overline{\mathcal{N}}_{L \alpha}^{i}\right)+8 \gamma_{\alpha}, \\
& \sum_{\alpha} R_{\alpha}^{3}=1 \bmod 2, \quad \text { with } R_{\alpha}^{3}=q_{s h \alpha}^{3}-\mathcal{N}_{L \alpha}^{3}+\overline{\mathcal{N}}_{L \alpha}^{3} .
\end{aligned}
$$


We can write down the rules for the $T^{6} / \mathbb{Z}_{12-I}$ orbifold on $\mathrm{SU}(3) \times F_{4}$ and $T^{6} / \mathbb{Z}_{12-I I}$ on $F_{4} \times \mathrm{SO}(4)$ analogously.

Now let us consider the additional $\mathbb{Z}_{2}$ Lorentz symmetry, generated by $\left(\theta_{1}\right)^{2}$, which we observed in subsection 3.1 for the $\mathbb{Z}_{4}$ orbifold on $\mathrm{SU}(4) \times \mathrm{SU}(4)$ and the $\mathbb{Z}_{8-I}$ orbifold on $\mathrm{SO}(5) \times \mathrm{SO}(9)$. In detail, this $\mathbb{Z}_{2}$ acts in the first complex plane, and leaves all the fixed points invariant up to lattice shifts. Consequently, the worldsheet instanton solutions for each auxiliary correlation function will enjoy the same symmetry, and this leads to an Rcharge conservation law. Then, we can write the selection rules for these orbifolds as follows.

$T^{6} / \mathbb{Z}_{4}$ on SU(4) $\times \mathrm{SU}(4)$ lattice. The torus lattice and orbifold twist cannot be factorized onto the three complex planes in this example. Therefore, a Q-charge conservation law emerges from the non-prime orbifold twist:

$$
\sum_{\alpha} Q_{\alpha}=0 \bmod 4, \quad \text { with } Q_{\alpha}=\sum_{i=1}^{3} 4 v^{i}\left(q_{s h \alpha}^{i}-\mathcal{N}_{L \alpha}^{i}+\overline{\mathcal{N}}_{L \alpha}^{i}\right)+4 \gamma_{\alpha} .
$$

Meanwhile, the additional $\mathbb{Z}_{2}$ symmetry, generated by $\left(\theta_{1}\right)^{2}$, enjoyed by all the fixed points leads to an R-charge conservation law:

$$
\sum_{\alpha} R_{\alpha}^{1}=1 \bmod 2, \quad \text { with } R_{\alpha}^{1}=\left(q_{s h \alpha}^{1}-\mathcal{N}_{L \alpha}^{1}+\overline{\mathcal{N}}_{L \alpha}^{1}\right)
$$

$T^{6} / \mathbb{Z}_{8-I}$ on $\mathrm{SO}(5) \times \mathrm{SO}(9)$ lattice. Here, although the torus lattice and orbifold twist can be factorized in the first complex plane, as the twist in that plane is non-prime, the correlation function does not factorize. Therefore, a Q-charge conservation law emerges from the orbifold twist:

$$
\sum_{\alpha} Q_{\alpha}=0 \bmod 8, \quad \text { with } Q_{\alpha}=\sum_{i=1}^{3} 8 v^{i}\left(q_{s h \alpha}^{i}-\mathcal{N}_{L \alpha}^{i}+\overline{\mathcal{N}}_{L \alpha}^{i}\right)+8 \gamma_{\alpha} .
$$

Again, the additional $\mathbb{Z}_{2}$ symmetry generated by $\left(\theta_{1}\right)^{2}$ leads to an R-charge conservation law:

$$
\sum_{\alpha} R_{\alpha}^{1}=1 \bmod 2, \quad \text { with } R_{\alpha}^{1}=q_{s h \alpha}^{1}-\mathcal{N}_{L \alpha}^{1}+\overline{\mathcal{N}}_{L \alpha}^{1}
$$

\section{Rule 4 in factorizable and non-factorizable orbifolds}

Finally, we should reconsider Rule 4 for the factorizable and non-factorizable, prime and non-prime orbifolds. The usual statement of Rule 4 for factorizable orbifolds is as follows [14-16]. When all the twisted states in a coupling lie at the same fixed point in a given plane, the symmetries relating the worldsheet instanton solutions are the full torus lattice automorphisms, which may be larger than the orbifold twist $\mathrm{N}^{i}$. This leads to an additional constraint for the non-vanishing couplings:

$$
\sum_{\alpha} \mathcal{N}_{L \alpha}^{i}-\overline{\mathcal{N}}_{L \alpha}^{i}-\overline{\mathcal{N}}_{R \alpha}^{i}=0 \bmod \mathrm{M}^{i}
$$


where $\mathrm{M}^{i}$ is the order of the torus lattice $\mathbb{Z}_{\mathrm{M}^{i}}$ automorphism group in the $i$-th plane. In the light of our previous discussion, this version of Rule 4 holds for factorizable prime planes. For non-prime planes in factorizable orbifolds, it must be checked that the twisted fields are at the same fixed point in all the auxiliary couplings allowed by the space group selection rule. In that case, every auxiliary coupling has the $\mathbb{Z}_{\mathrm{M}^{i}}$ symmetry in the $i$-th plane, so Rule 4 again applies as above, and is generally stronger than the R-charge conservation condition (3.45), even when the order of the torus lattice automorphisms matches that of the point group.

For the non-factorizable orbifolds, when all twisted fields are at the same fixed point in each allowed auxiliary coupling, 6D torus (sub)lattice automorphisms such as the orbifold twist $^{8} \theta$, are observed in each auxiliary correlation function. Thus, we are lead to the condition (for all fields at the same fixed point in all auxiliary correlation functions):

$$
\sum_{i=1}^{3} \sum_{\alpha} \mathrm{N} v^{i}\left(\mathcal{N}_{L \alpha}^{i}-\overline{\mathcal{N}}_{L \alpha}^{i}-\overline{\mathcal{N}}_{R \alpha}^{i}\right)=0 \bmod \mathrm{N},
$$

which is independent of the orbifold projection condition for non-prime orbifolds, and hence constitutes a non-trivial selection rule there.

Moreover, every torus lattice has a $\mathbb{Z}_{2}$ symmetry, that is, the lattice vectors always come in pairs $\{\lambda,-\lambda\}$. Thus we obtain the additional condition (again, for all fields at the same fixed point in all auxiliary correlation functions) ${ }^{9}$

$$
\sum_{i=1}^{3} \sum_{\alpha}\left(\mathcal{N}_{L \alpha}^{i}-\overline{\mathcal{N}}_{L \alpha}^{i}-\overline{\mathcal{N}}_{R \alpha}^{i}\right)=0 \bmod 2 .
$$

For example, for the $\mathbb{Z}_{7}$ orbifold on an $\mathrm{SU}(7)$ root lattice, we have

$$
\sum_{i=1}^{3} \sum_{\alpha}\left(\mathcal{N}_{L \alpha}^{i}-\overline{\mathcal{N}}_{L \alpha}^{i}-\overline{\mathcal{N}}_{R \alpha}^{i}\right)=0 \bmod 2,
$$

whereas for a $\mathbb{Z}_{6-I I}$ orbifold on an $\mathrm{SU}(6) \times \mathrm{SU}(2)$ lattice we have:

$$
\begin{aligned}
& \sum_{i=1}^{3} \sum_{\alpha} 6 v^{i}\left(\mathcal{N}_{L \alpha}^{i}-\overline{\mathcal{N}}_{L \alpha}^{i}-\overline{\mathcal{N}}_{R \alpha}^{i}\right)=0 \bmod 6 \\
& \sum_{i=1}^{3} \sum_{\alpha}\left(\mathcal{N}_{L \alpha}^{i}-\overline{\mathcal{N}}_{L \alpha}^{i}-\overline{\mathcal{N}}_{R \alpha}^{i}\right)=0 \bmod 2 .
\end{aligned}
$$

Finally, we should note that as Rule 4 applies only to some couplings, in fact depending on the relative distance between the twisted strings in the orbifold space, it cannot be interpreted as a conventional symmetry in the low energy effective field theory [17].

\footnotetext{
${ }^{8}$ In contrast, for couplings involving twisted fields at different fixed points in non-prime orbifolds, the orbifold twist symmetry is observed in the correlation function only after putting all the auxiliary correlation functions together. The consequences of this symmetry were discussed in section 3.3.2.

${ }^{9}$ Note that the torus lattice has the $\mathbb{Z}_{\mathrm{N}}$ symmetry and the $\mathbb{Z}_{2}$ symmetry, but this does not necessarily imply it has a $\mathbb{Z}_{2 \mathrm{~N}}$ symmetry nor that $\mathbb{Z}_{2} \subset \mathbb{Z}_{\mathrm{N}}$ for $\mathrm{N}$ even!
} 


\section{Conclusions}

In this paper we have derived string coupling selection rules for $T^{6} / \mathbb{Z}_{\mathrm{N}}$ orbifolds. Our method builds on that used in [13], but we now consider the various classes of orbifolds, with the torus lattice $\Lambda$ factorizable and non-factorizable, and the orbifold twist $\theta$ of prime and non-prime order. We have found corrections to the old R-charge conservation rule for factorizable orbifolds, studied selection rules for non-factorizable orbifolds, and identified a new stringy selection rule that we call Rule 6 .

Couplings between twisted strings generically have classical contributions from worldsheet instantons stretching between the fixed points and wrapping the orbifold geometry. In computing the coupling strength, or corresponding correlation function, we have to sum over all such possible classical solutions. The set of possible instanton solutions, which are proportional to vectors lying on particular cosets of the torus lattice, enjoys some symmetry. This symmetry often leads some couplings to vanish. In particular, we can write down some instanton selection rules, which turn out to constrain the number of oscillators that can appear in non-vanishing couplings.

One of these instanton selection rules is the well-known R-charge conservation law. Actually, R-charge conservation is usually understood in terms of discrete Lorentz symmetries that survive the orbifolding. In particular, in [14-17], it was understood that the factorizable $T^{6} / \mathbb{Z}_{3}$ orbifold's geometry is invariant under the orbifold twist acting independently in each of the planes. It turns out that the allowed couplings are similarly invariant under such independent twists, which correspond to symmetries among the set of worldsheet instantons. Thus one is lead to a twist invariance condition, which, put together with H-momentum conservation, leads to a conserved R-charge. In this way, discrete Lorentz symmetries in the orbifold geometry, which distinguish between bosons and fermions, lead naturally to discrete R-symmetries in the low energy effective field theory describing the compactification.

This intuition was subsequently applied to all factorizable orbifolds. Hence the current consensus is that in factorizable orbifolds, there is a twist symmetry acting independently plane by plane, which leads to an R-charge conservation condition in each of the three planes, $\sum_{\alpha} R_{\alpha}^{i}=1 \bmod \mathrm{N}^{i}$, with R-charges given by $R_{\alpha}^{i}=q_{s h \alpha}^{i}-\mathcal{N}_{L \alpha}^{i}+\overline{\mathcal{N}}_{L \alpha}^{i}$ and $\mathrm{N}^{i}$ the order of the orbifold twist in the $i$-th plane. Then it remained to understand possible R-charge conservation laws in non-factorizable orbifolds. By carefully considering the derivation of the R-charge conservation rule from the orbifold CFT, however, we find the old R-charge conservation law to hold only for the prime, factorizable orbifold.

More generally, one has to take care when assigning the conserved R-charges to the string states. The string orbifold compactification includes twisted sectors, which are localized at the fixed point singularities and emerge only due to the compactification. As a consequence, intuition gained from field theoretic smooth compactifications has to be checked in string orbifold compactifications. Indeed, it is not clear how the twisted states transform under the orbifold twist acting independently in each plane. Therefore, to identify the R-charge conservation law for allowed couplings, we have to consider the consequences of the orbifold's geometrical symmetries in the correlation functions, and in particular in the worldsheet instanton solutions. 
For non-prime orbifolds, the higher twisted sectors are attached to fixed points that are not necessarily $\theta$-invariant. Physical states are then built up using linear combinations of twisted strings attached to different fixed points in the same conjugacy class, whose constant coefficients are powers of $e^{-2 \pi \imath \gamma}$, with $\gamma$ the $\gamma$-phase [37]. The geometrical part of the state is then a $\theta$-eigenstate, with eigenvalue $e^{2 \pi \imath \gamma}$, and only the complete state is $\theta$-invariant. Correlation functions between physical states are then sums of auxiliary correlation functions between states at particular fixed points, weighted by the $\gamma$-phases. Consequently, the sum over instanton solutions is also weighted by the $\gamma$-phases. The twist relations among the fixed points do lead to symmetries among the instanton solutions, and these symmetries in the geometry do lead to R-charge conservation laws, but the conserved R-charges are different to those found in the literature. In particular, the $\gamma$ phase contributes non-trivially to the charges, and an independent R-charge conservation law is obtained only for prime planes in factorizable orbifolds, whereas non-prime planes all contribute to a single R-charge conservation.

At this point, it may be useful to recall the rather convoluted history of the $\gamma$-phase in orbifold selection rules. For several years it was believed that non-vanishing couplings must satisfy the so-called $\gamma$-rule, which stated that the sum of the $\gamma$-phases must be trivial $[18$, 36]. Then, in [47] it was emphasised that the $\gamma$-rule is in fact automatically satisfied by all couplings between physical states, after imposing gauge invariance and the R-charge conservation rule. Careful derivation of the selection rules from the CFT tells a different story. R-charge conservation and H-momentum conservation constrain the sum of the $\gamma$-phases to be trivial for three-point couplings between massless ground states, that is, couplings that do not involve oscillators. However, there is no independent $\gamma$-rule, and in general R-charge conservation poses weaker constraints on the $\gamma$-phase.

A similar analysis can be made for non-factorizable orbifolds. As the twist does not act plane by plane, there are in general no independent twist symmetries. For prime orbifolds, the $6 \mathrm{D}$ twist symmetry that relates the worldsheet instanton solutions is automatically satisfied for all possible couplings, and thus there is no corresponding selection rule. For non-prime orbifolds, the weighting of the worldsheet instanton solutions by the $\gamma$-phases in the correlation function leads to a single charge conservation law, where again, the $\gamma$-phases contribute to the charges, and now the corresponding symmetry is one that commutes with supersymmetry. Moreover, in some special cases, the worldsheet instantons for nonfactorizable orbifolds enjoy a further $\mathbb{Z}_{2}$ symmetry in one complex plane, which leads to an R-charge conservation law.

Additional symmetries in the worldsheet instanton solutions can lead to more selection rules, which constrain the oscillator numbers. An example of this is already known; Rule 4 or the torus lattice selection rule [13-16]. This occurs for couplings with all twisted fields at the same fixed point, in which case the symmetry among the worldsheet instanton solutions is enhanced from the twist symmetry to the full torus lattice symmetries. This applies, in different forms, for factorizable and non-factorizable, prime and non-prime orbifolds. We have also found another such rule for the factorizable non-prime orbifolds, with two nonprime planes, which we may call Rule 6 or the coset vector selection rule. When the lowest common multiple, $\mathrm{K}$, of the twisted sectors in a given coupling is less than the order $\mathrm{N}$ of 
the point group, the worldsheet instanton solutions enjoy an additional symmetry, of order $\mathrm{N} / \mathrm{K}$. We summarize our results with table 3 . In table 4 , we compare the number of allowed trilinear couplings in a $T^{6} / Z_{6-I I}$ model, ${ }^{10}$ applying the presently used selection rules with old R-charges, the presently used selection rules with correct R-charges, and the presently used selection rules with correct R-charges plus Rules 4, 5 and 6 . Observe that many couplings that were ruled out by the old R-charge conservation law are actually allowed.

The question that comes to the mind of all Orbifolders is now: what is the relevance of the string selection rules for phenomenology? We expect the charge conservation laws to be associated with symmetries in the low energy effective field theory. However, such an interpretation seems more challenging for Rules 4 and 6 , as they apply only to some kinds of couplings, depending on the relative properties of the participating twisted strings. Thus, intriguingly, it would seem that Rules 4 and 6 correspond to "stringy miracles" from the point of view of a $4 \mathrm{D}$ observer. The same can be said for Rule 5 .

So far, we have only considered models without discrete Wilson lines, whereas all promising models use discrete Wilson lines to reduce the gauge group and the number of generations. In the presence of discrete Wilson lines, new so-called shift $\gamma$-phases appear $[19,20,49]$. It would be important to work out their role in the string couplings.

Meanwhile, the selection rules we have computed are for couplings in the holomorphic superpotential of the low energy effective field theory. After moduli stabilization, an effective superpotential emerges, which also has contributions from the Kähler potential. In $[13,50]$, one can find a special case of an effectively holomorphic matter coupling that is allowed in the effective superpotential, but forbidden by Rules 4 and 5 in the truely holomorphic superpotential. Without a better understanding of the Kähler potential [51], we cannot write down the allowed couplings in the effective superpotential.

Another interesting issue is to study anomalies for the R-symmetries that we have found. Such a study on R-symmetry anomalies has been performed for the standard Rcharge conservation law in factorizable orbifolds in [52]. It would be important to reconsider such studies taking into account our results such as the inclusion of the $\gamma$ phases. We would also like to understand further the role of selection rules in the matching between orbifold compactifications and their Calabi-Yau blowups [53, 54].

We close our discussion by writing down an algorithmn to compute the allowed Lpoint couplings for a $\mathbb{Z}_{\mathrm{N}}$ orbifold. We present here the selection rules for the factorizable orbifolds, but the non-factorizable case follows analogously from the main part of the paper and table 3. The rules can be applied as follows:

1. Gauge invariance: $\sum_{\alpha} p_{s h}=0$.

2. Space group selection rule: $\prod_{\alpha}\left[\left(\theta^{k_{\alpha}}, \lambda_{\alpha}\right)\right]=(\mathbb{1}, 0)$.

3. H-momentum conservation: This does not restrict the allowed couplings, but allows one to determine the right-moving oscillator numbers as $\overline{\mathcal{N}}_{R}^{i}=\sum_{\alpha} q_{s h \alpha}^{i}-1$.

\footnotetext{
${ }^{10}$ We computed the spectrum of this model using the orbifolder [48]. Then we developed a code to compute the couplings implementing Rule 4, Rule 5, Rule 6 and the correct R-charge selection rules.
} 


\begin{tabular}{|l|l|}
\hline & $\mathrm{R} / \mathrm{Q}$-charge Conservation \\
\hline Factorizable, prime & $\sum_{\alpha} R_{\alpha}^{i}=1 \bmod \mathrm{N}^{i}, \quad i=1,2,3$ \\
& with $R_{\alpha}^{i}=q_{s h \alpha}^{i}-\mathcal{N}_{L \alpha}^{i}+\overline{\mathcal{N}}_{L \alpha}^{i}$ \\
\hline Factorizable, non-prime & $\sum_{\alpha} R_{\alpha}^{j}=1 \bmod \mathrm{N}^{j}, \quad j=$ prime planes \\
& with $R_{\alpha}^{j}=q_{s h \alpha}^{j}-\mathcal{N}_{L \alpha}^{j}+\overline{\mathcal{N}}_{L \alpha}^{j}$, \\
& $\sum_{\alpha} R_{\alpha}=\left(\sum_{i \neq j} \mathrm{~N} v^{i}\right) \bmod \mathrm{N}$ \\
& with $R_{\alpha}=\sum_{i \neq j} \mathrm{~N} v^{i}\left(q_{s h \alpha}^{i}-\mathcal{N}_{L \alpha}^{i}+\overline{\mathcal{N}}_{L \alpha}^{i}\right)+\mathrm{N} \gamma_{\alpha}$ \\
\hline Non-factorizable, prime & none \\
\hline Non-factorizable, non-prime & $\sum_{\alpha} Q_{\alpha}=0 \bmod \mathrm{N}$ \\
& with $Q_{\alpha}=\sum_{i=1}^{3} \mathrm{~N} v^{i}\left(q_{s h \alpha}^{i}-\mathcal{N}_{L \alpha}^{i}+\overline{\mathcal{N}}_{L \alpha}^{i}\right)+\mathrm{N} \gamma_{\alpha}$, \\
& $\sum_{\alpha} R_{\alpha}^{i}=1 \bmod \mathrm{N}^{i} / n, \quad$ for $\left(\theta_{i}\right)^{n}$ automorphism \\
& with $R_{\alpha}^{i}=q_{s h \alpha}^{i}-\mathcal{N}_{L \alpha}^{i}+\overline{\mathcal{N}}_{L \alpha}^{i}$ \\
\hline
\end{tabular}

\begin{tabular}{|c|l|}
\hline & Rule 4 for all twisted sectors at same fixed point \\
\hline Factorizable, prime & $\mathcal{N}_{L}^{i}-\overline{\mathcal{N}}_{L}^{i}-\overline{\mathcal{N}}_{R}^{i}=0 \bmod 2 \mathrm{~N}^{i}, \quad i=1,2,3$ \\
\hline Factorizable, non-prime & $\mathcal{N}_{L}^{i}-\overline{\mathcal{N}}_{L}^{i}-\overline{\mathcal{N}}_{R}^{i}=0 \bmod \mathrm{N}^{i}, \quad i=1,2,3$ \\
\hline Non-factorizable, prime & $\sum_{i=1}^{3}\left(\mathcal{N}_{L}^{i}-\overline{\mathcal{N}}_{L}^{i}-\overline{\mathcal{N}}_{R}^{i}\right)=0 \bmod 2$ \\
\hline Non-factorizable, non-prime & $\sum_{i=1}^{3} \mathrm{~N} v^{i}\left(\mathcal{N}_{L}^{i}-\overline{\mathcal{N}}_{L}^{i}-\overline{\mathcal{N}}_{R}^{i}\right)=0 \bmod \mathrm{N}$ \\
& $\sum_{i=1}^{3}\left(\mathcal{N}_{L}^{i}-\overline{\mathcal{N}}_{L}^{i}-\overline{\mathcal{N}}_{R}^{i}\right)=0 \bmod 2$ \\
\hline
\end{tabular}

\begin{tabular}{|c|l|}
\hline & Rule 6 with $\mathrm{K}=\operatorname{lcm}\left(l_{1}, \ldots, l_{\mathrm{L}}\right)$ \\
\hline Factorizable, prime & none \\
\hline Factorizable, non-prime & $\mathcal{N}_{L}^{i}-\overline{\mathcal{N}}_{L}^{i}-\overline{\mathcal{N}}_{R}^{i}=0 \bmod \mathrm{N}^{i} / \mathrm{K}, \quad$ for two non-prime $i$ \\
\hline Non-factorizable, prime & none \\
\hline Non-factorizable, non-prime & none \\
\hline
\end{tabular}

Table 3. R/Q-charge conservation, Rule 4 and Rule 6 for the various $T^{6} / \mathbb{Z}_{\mathrm{N}}$ orbifolds, with $T^{6}$ factorizable or non-factorizable, and $\mathrm{N}$ prime or non-prime. We write the order of the orbifold twist in plane $i$ as $\mathrm{N}^{i}$. For $\mathrm{N}$ non-prime, $\mathrm{N}^{i}$ may be prime or non-prime. We use $\mathcal{N}_{L \alpha}, \overline{\mathcal{N}}_{L \alpha}$ for the oscillator numbers of each state, and $\mathcal{N}_{L}, \overline{\mathcal{N}}_{L}, \overline{\mathcal{N}}_{R}$ for the total number of oscillators in the correlation function. The fixed points to which the twisted states are attached satisfy $\theta^{l_{\alpha}} f_{\alpha}=f_{\alpha}+\lambda$, with $l_{\alpha}$ the smallest such integer, and $l \mathrm{~cm}$ stands for lowest common multiple. Untwisted states may also participate in the couplings. The rules for the partially factorizable orbifolds are similar, and can be found explicitly in the text.

4. Rule 5 or forbidden instanton selection rule: Check whether holomorphic and antiholomorphic instantons in the $i$-th plane are allowed. Non-trivial holomorphic solutions exist if and only if $1+\sum_{\alpha}\left(-1+\mathbf{k}_{\alpha}^{i}\right)<0$ (where $0<\mathbf{k}_{\alpha}^{i} \leq 1$ ). Non-trivial antiholomorphic solutions exist if and only if $1+\sum_{\alpha}\left(-\mathbf{k}_{\alpha}^{i}\right)<0$ (where $0 \leq \mathbf{k}_{\alpha}^{i}<1$ ). If 


\begin{tabular}{|l|c|c|c|}
\hline & Used Rules & Correct R-charges & All Rules \\
\hline Number of couplings & 96 & 156 & 132 \\
\hline
\end{tabular}

Table 4. Comparison of the number of allowed trilinear couplings between twisted fields in a $T^{6} / Z_{6-I I}$ model, applying the presently used selection rules (gauge invariance, the space group selection rule and R-charge conservation) with old R-charges, the presently used selection rules with correct R-charges, and the presently used selection rules with correct R-charges plus Rules 4, 5 and 6 (Rule 6 is in fact not independent for $T^{6} / Z_{6-I I}$ ). The gauge shift vector defining the model is $V^{I}=\left(\frac{1}{3},-\frac{1}{2},-\frac{1}{2}, 0,0,0,0,0, \frac{1}{2},-\frac{1}{6},-\frac{1}{2},-\frac{1}{2},-\frac{1}{2},-\frac{1}{2},-\frac{1}{2}, \frac{1}{2}\right)$, with gauge group $\mathrm{SO}(10) \times \mathrm{SU}(2) \times$ $\mathrm{SU}(2) \times \mathrm{SO}(14) \times \mathrm{U}(1)^{2}$.

both holomorphic and anti-holomorphic solutions are vanishing, then we require $\mathcal{N}_{L}^{i}=$ $\overline{\mathcal{N}}_{L}^{i}+\overline{\mathcal{N}}_{R}^{i}$. If holomorphic instantons are allowed, but anti-holomorphic instantons are vanishing, then $\mathcal{N}_{L}^{i} \geq \overline{\mathcal{N}}_{L}^{i}$. If instead only anti-holomorphic instantons are allowed, then $\mathcal{N}_{L}^{i} \leq \overline{\mathcal{N}}_{L}^{i}+\overline{\mathcal{N}}_{R}^{i}$

5. R-charge conservation: Applies when instanton solutions are allowed in the plane $i$. For planes $j$ with prime ordered twist $\mathrm{N}^{j}$, we have $\mathcal{N}_{L}^{j}-\overline{\mathcal{N}}_{L}^{j}-\overline{\mathcal{N}}_{R}^{j}=0 \bmod \mathrm{N}^{j}$. For non-prime planes, we have $\sum_{i \neq j} \mathrm{~N} v^{i}\left(\mathcal{N}_{L}^{i}-\overline{\mathcal{N}}_{L}^{i}-\overline{\mathcal{N}}_{R}^{i}\right)-\mathrm{N} \sum_{\alpha} \gamma_{\alpha}=0 \bmod \mathrm{N}$.

6. Rule 6 or coset vector selection rule: Applies when two planes $i \neq j$ are non-prime, and when instanton solutions are allowed in the non-prime planes. For couplings with $\mathrm{K}<\mathrm{N}$, for $\mathrm{K}$ the lowest common multiple of the twisted sectors (or their conjugates), we have $\mathcal{N}_{L}^{i}-\overline{\mathcal{N}}_{L}^{i}-\overline{\mathcal{N}}_{R}^{i}=0 \bmod \frac{\mathrm{N}^{i}}{\mathrm{~K}}$.

7. Rule 4 or torus lattice selection rule: Applies when instanton solutions are allowed in the planes $i$. When all twisted sectors are at the same fixed point in plane $i$ for every auxiliary coupling, we have $\mathcal{N}_{L}^{i}-\overline{\mathcal{N}}_{L}^{i}-\overline{\mathcal{N}}_{R}^{i}=0 \bmod \mathrm{M}^{i}$ where $\mathrm{M}^{i}$ is the order of the torus lattice automorphism group in plane $i$.

\section{Acknowledgments}

We would like to thank M. Blaszczyk, S. Förste, C. Lüdeling, P. Oehlmann, N. Pagani, S. Ramos-Sánchez, F. Rühle and P. Vaudrevange for useful discussions. We also thank the organizers of the 4th Bethe Center Workshop and Bethe Forum, Bad Honnef, Germany for kind hospitality while part of this work was done. D. M. P. and M. S. would also like to thank the Simons Center for Geometry and Physics and the organizers of the Summer School on String Phenomenology in Stony Brook (NY) where part of this work was done. N. G. C. B. is partially supported by "Centro de Aplicaciones Tecnológicas y Desarrollo Nuclear" (CEADEN), "Proyecto Nacional de Ciencias Básicas Partículas y Campos" (CITMA, Cuba) and the European Commission under the contract PITN-GA-2009-237920 during her stay at CERN. T. K. is supported in part by the Grant-in-Aid for the Global COE Program "The Next Generation of Physics, Spun from Universality and Emergence" from the Ministry of Education, Culture, Sports, Science and Technology of Japan. S. L. P. is funded by Deutsche Forschungsgemeinschaft inside the "Graduiertenkolleg GRK 1463". The work 
of N. G. C. B., D. M. P. and M. S. was partially supported by the SFB-Tansregio TR33 "The Dark Universe" (Deutsche Forschungsgemeinschaft) and the European Union 7th network program "Unification in the LHC era" (PITN-GA-2009-237920).

\section{A A classification of orbifold automorphisms}

In this appendix we present a more general discussion of the orbifold automorphisms. As we saw in the paper, a crucial point is that the instanton solutions are related to the fixed points by means of the coset vectors. We are thus interested in symmetries that respect the fixed points of the orbifold. Since the symmetries of the fixed points can be related to the lattice automorphisms, we take this group as the starting point of our exploration. This discrete group is a subgroup of the Lorentz group in the compact six dimensional space. Given that the Lorentz symmetry is the only one which makes a distinction between bosons and fermions, the members of $\operatorname{Aut}(\Lambda)$ which happen to survive the orbifold identifications seem suitable to explain the presence of discrete $R$-symmetries in the low energy effective theory.

In order to classify the elements of $\operatorname{Aut}(\Lambda)$, we define certain subgroups, as displayed in figure 2. The biggest subgroup, $A \subset \operatorname{Aut}(\Lambda)$ is the group of automorphisms that respect the point group $P$,

$$
A=\left\{\varrho \in \operatorname{Aut}(\Gamma) \mid \varrho \theta^{k} \varrho^{-1} \in P, \forall \theta^{k} \in P\right\} .
$$

Clearly any vector in the compact dimensions will transform under the elements of Aut $(\Lambda)$. Consequently the action of any $\varrho \in A$ on a space group element $h=\left(\theta^{k}, \lambda\right) \in S$ is given by

$$
\varrho(h)=\left(\varrho \theta^{k} \varrho^{-1}, \varrho \lambda\right) .
$$

Note that $A$ is constructed in such a way that it preserves the structure of the conjugacy classes. That is, given two space group elements $g_{1}, g_{2}$ which belong to the same conjugacy class $[g]$, any transformation $\varrho \in A$ will preserve the network of identifications, i.e. $\varrho\left(g_{1}\right) \sim$ $\varrho\left(g_{2}\right) \in[\varrho(g)]$. We define further the subgroups

$$
\begin{aligned}
& B=\left\{\varrho \in A \mid\left[\varrho, \theta^{k}\right]=0, \forall \theta^{k} \in P\right\} \\
& C=\left\{\varrho \in B \mid \forall z_{f} \text { fixed point of } S, \exists h \in S \text { s.t. } \varrho z_{f}=h z_{f}\right\}, \\
& D=\{\varrho \in C \mid \operatorname{det}(\varrho)=1\}
\end{aligned}
$$

where $B$ is the subgroup of symmetries which map between conjugacy classes of the same twisted sector. $C$ is defined as the subgroup of automorphisms which preserve all the conjugacy classes of the space group, i.e. $[\varrho(g)]=[g]$, and $D$ contains all elements in $C$ which belong to $\mathrm{SO}(6)$. It is easy to show, that these subgroups fulfill

$$
D \triangleleft C \triangleleft B \triangleleft A,
$$

and hence it makes sense to define the corresponding quotient groups $E=C / D, F=B / C$ and $G=A / B$. 


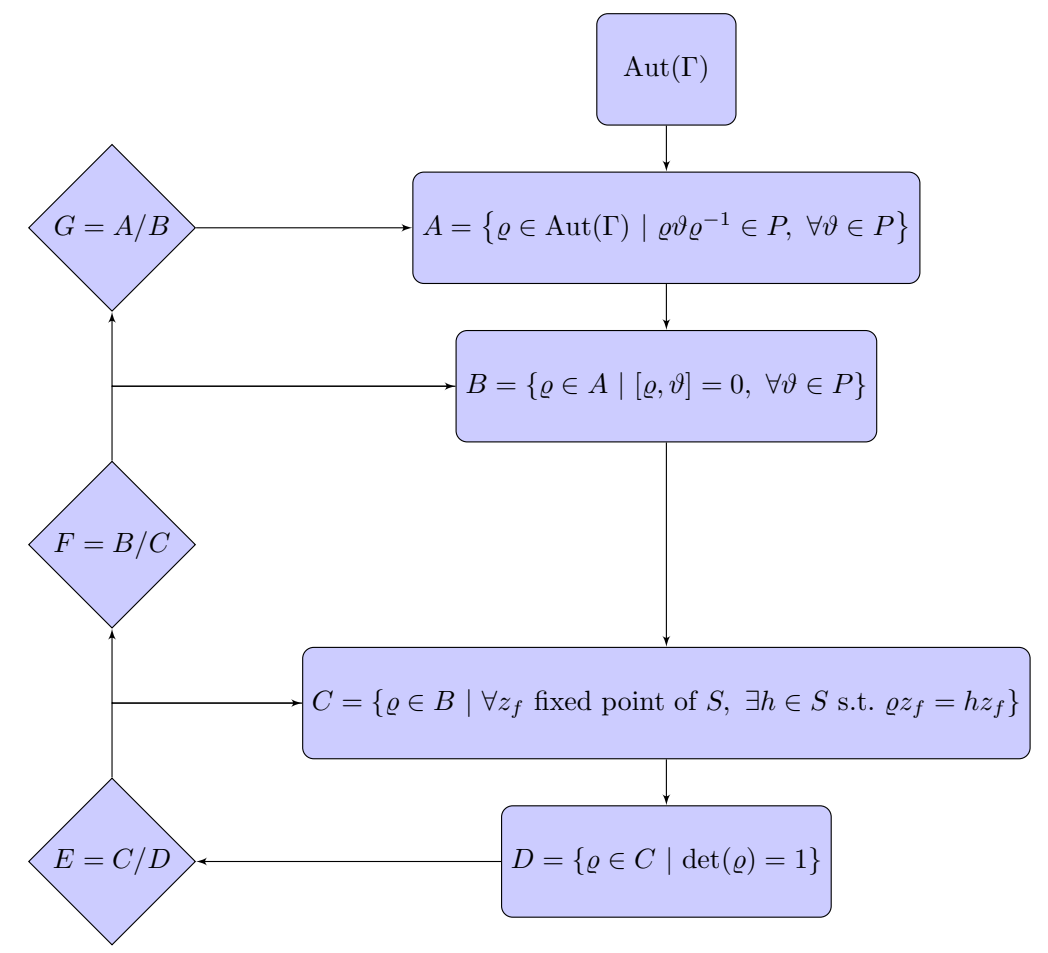

Figure 2. The subgroup $A$ of the automorphism group of the six dimensional lattice allows for a decomposition into the subgroups $B, C$ and $D$, as defined in eqs. (A.3), (A.4) and (A.5). Provided the normalcy relations between them, one can construct the quotients $E, F$ and $G$ which allow for a simpler interpretation given their reduced number of elements.

The elements in $G=A / B$ are symmetries which exchange space group elements of different twisted sectors, whereas the elements of $F=B / C$ map between inequivalent fixed points within the same twisted sector. The group $E=C / D$ contains all reflections in $O(6)$ which commute with the point group and map all conjugacy classes of the space group to themselves. These quotient groups are very interesting objects to study but the discussion of their implications in the string theory is beyond the scope of this work. In the main text we restricted to elements of group $D$ because they commute with the point group, leave all fixed point conjugacy classes invariant and can be written in terms of the Cartan generators of $\mathrm{SO}(6)$.

Open Access. This article is distributed under the terms of the Creative Commons Attribution License which permits any use, distribution and reproduction in any medium, provided the original author(s) and source are credited.

\section{References}

[1] L.B. Anderson, J. Gray, A. Lukas and E. Palti, Two hundred heterotic standard models on smooth Calabi-Yau threefolds, Phys. Rev. D 84 (2011) 106005 [arXiv:1106.4804] [INSPIRE].

[2] O. Lebedev et al., A mini-landscape of exact MSSM spectra in heterotic orbifolds, Phys. Lett. B 645 (2007) 88 [hep-th/0611095] [INSPIRE]. 
[3] P. Anastasopoulos, T. Dijkstra, E. Kiritsis and A. Schellekens, Orientifolds, hypercharge embeddings and the standard model, Nucl. Phys. B 759 (2006) 83 [hep-th/0605226] [INSPIRE].

[4] K.R. Dienes, M. Lennek, D. Senechal and V. Wasnik, Supersymmetry versus gauge symmetry on the heterotic landscape, Phys. Rev. D 75 (2007) 126005 [arXiv:0704.1320] [InSPIRE].

[5] F. Gmeiner, R. Blumenhagen, G. Honecker, D. Lüst and T. Weigand, One in a billion: MSSM-like D-brane statistics, JHEP 01 (2006) 004 [hep-th/0510170] [INSPIRE].

[6] C. Beasley, J.J. Heckman and C. Vafa, GUTs and exceptional branes in F-theory - II: experimental predictions, JHEP 01 (2009) 059 [arXiv:0806.0102] [INSPIRE].

[7] S. Raby, Searching for the standard model in the string landscape: SUSY GUTs, Rept. Prog. Phys. 74 (2011) 036901 [arXiv: 1101.2457] [INSPIRE].

[8] A.E. Faraggi, MSHSM - Minimal Standard Heterotic String Models, Fortsch. Phys. 58 (2010) 733 [arXiv:1002.0052] [INSPIRE].

[9] B. Gato-Rivera and A. Schellekens, Asymmetric Gepner models: revisited, Nucl. Phys. B 841 (2010) 100 [arXiv: 1003.6075] [INSPIRE].

[10] A. Maharana and E. Palti, Models of particle physics from type IIB string theory and F-theory: a review, arXiv: 1212.0555 [INSPIRE].

[11] L.J. Dixon, D. Friedan, E.J. Martinec and S.H. Shenker, The conformal field theory of orbifolds, Nucl. Phys. B 282 (1987) 13 [INSPIRE].

[12] S. Hamidi and C. Vafa, Interactions on orbifolds, Nucl. Phys. B 279 (1987) 465 [INSPIRE].

[13] T. Kobayashi, S.L. Parameswaran, S. Ramos-Sanchez and I. Zavala, Revisiting coupling selection rules in heterotic orbifold models, JHEP 05 (2012) 008 [Erratum ibid. 12 (2012) 049] [arXiv: 1107.2137] [INSPIRE].

[14] A. Font, L.E. Ibáñez, H.P. Nilles and F. Quevedo, Degenerate orbifolds, Nucl. Phys. B 307 (1988) 109 [Erratum ibid. B 310 (1988) 764] [INSPIRE].

[15] A. Font, L. E. Ibáñez, H. P. Nilles and F. Quevedo, Yukawa couplings in degenerate orbifolds: towards a realistic $\mathrm{SU}(3) \times \mathrm{SU}(2) \times \mathrm{U}(1)$ superstring, Phys. Lett. B 210 (1988) 101 [Erratum ibid. B 213 (1988) 564] [INSPIRE].

[16] A. Font, L.E. Ibáñez, F. Quevedo and A. Sierra, The construction of 'realistic' four-dimensional strings through orbifolds, Nucl. Phys. B 331 (1990) 421 [INSPIRE].

[17] A. Font, L.E. Ibáñez, H.P. Nilles and F. Quevedo, On the concept of naturalness in string theories, Phys. Lett. B 213 (1988) 274 [INSPIRE].

[18] T. Kobayashi, S. Raby and R.-J. Zhang, Searching for realistic $4 D$ string models with a Pati-Salam symmetry: orbifold grand unified theories from heterotic string compactification on a Z(6) orbifold, Nucl. Phys. B 704 (2005) 3 [hep-ph/0409098] [INSPIRE].

[19] P.K.S. Vaudrevange, Grand unification in the heterotic brane world, arXiv:0812.3503 [INSPIRE].

[20] S. Ramos-Sanchez, Towards low energy physics from the heterotic string, Fortsch. Phys. 10 (2009) 907 [arXiv:0812.3560] [INSPIRE].

[21] J. Erler, D. Jungnickel and J. Lauer, Dependence of Yukawa couplings on the axionic background moduli of $Z(N)$ orbifolds, Phys. Rev. D 45 (1992) 3651 [INSPIRE]. 
[22] T. Kobayashi and O. Lebedev, Heterotic Yukawa couplings and continuous Wilson lines, Phys. Lett. B 566 (2003) 164 [hep-th/0303009] [INSPIRE].

[23] T. Kobayashi and O. Lebedev, Heterotic string backgrounds and CP-violation, Phys. Lett. B 565 (2003) 193 [hep-th/0304212] [INSPIRE].

[24] T. Kobayashi, S. Raby and R.-J. Zhang, Constructing $5 D$ orbifold grand unified theories from heterotic strings, Phys. Lett. B 593 (2004) 262 [hep-ph/0403065] [INSPIRE].

[25] W. Buchmüller, K. Hamaguchi, O. Lebedev and M. Ratz, Supersymmetric standard model from the heterotic string, Phys. Rev. Lett. 96 (2006) 121602 [hep-ph/0511035] [INSPIRE].

[26] W. Buchmüller, K. Hamaguchi, O. Lebedev and M. Ratz, Supersymmetric standard model from the heterotic string (II), Nucl. Phys. B 785 (2007) 149 [hep-th/0606187] [INSPIRE].

[27] J.E. Kim and B. Kyae, Flipped SU(5) from Z(12-I) orbifold with Wilson line, Nucl. Phys. B 770 (2007) 47 [hep-th/0608086] [INSPIRE].

[28] J.E. Kim, J.-H. Kim and B. Kyae, Superstring standard model from Z(12-I) orbifold compactification with and without exotics and effective R-parity, JHEP 06 (2007) 034 [hep-ph/0702278] [INSPIRE].

[29] O. Lebedev, H.P. Nilles, S. Ramos-Sanchez, M. Ratz and P.K. Vaudrevange, Heterotic mini-landscape. II. Completing the search for MSSM vacua in a $Z(6)$ orbifold, Phys. Lett. B 668 (2008) 331 [arXiv:0807.4384] [INSPIRE].

[30] M. Blaszczyk et al., $A Z_{2} \times Z_{2}$ standard model, Phys. Lett. B 683 (2010) 340 [arXiv:0911.4905] [INSPIRE].

[31] L.J. Dixon, J.A. Harvey, C. Vafa and E. Witten, Strings on orbifolds, Nucl. Phys. B 261 (1985) 678 [INSPIRE].

[32] L.J. Dixon, J.A. Harvey, C. Vafa and E. Witten, Strings on orbifolds. 2, Nucl. Phys. B 274 (1986) 285 [INSPIRE].

[33] M. Fischer, M. Ratz, J. Torrado and P.K. Vaudrevange, Classification of symmetric toroidal orbifolds, JHEP 01 (2013) 084 [arXiv:1209.3906] [INSPIRE].

[34] Y. Katsuki et al., Z(N) orbifold models, Nucl. Phys. B 341 (1990) 611 [InSPIRE].

[35] T. Kobayashi and N. Ohtsubo, Geometrical aspects of $Z(N)$ orbifold phenomenology, Int. J. Mod. Phys. A 9 (1994) 87 [inSPIRE].

[36] J. Casas, F. Gómez and C. Muñoz, Complete structure of $Z(n)$ Yukawa couplings, Int. J. Mod. Phys. A 8 (1993) 455 [hep-th/9110060] [InSPIRE].

[37] T. Kobayashi and N. Ohtsubo, Yukawa coupling condition of $Z(n)$ orbifold models, Phys. Lett. B 245 (1990) 441 [inSPIRE].

[38] J. Lauer, J. Mas and H.P. Nilles, Twisted sector representations of discrete background symmetries for two-dimensional orbifolds, Nucl. Phys. B 351 (1991) 353 [INSPIRE].

[39] J. Erler, D. Jungnickel, J. Lauer and J. Mas, String emission from twisted sectors: cocycle operators and modular background symmetries, Annals Phys. 217 (1992) 318 [INSPIRE].

[40] M. Cvetič, Suppression of nonrenormalizable terms in the effective superpotential for (blownup) orbifold compactification, Phys. Rev. Lett. 59 (1987) 1795 [INSPIRE].

[41] J. Erler, D. Jungnickel, M. Spalinski and S. Stieberger, Higher twisted sector couplings of $Z(N)$ orbifolds, Nucl. Phys. B 397 (1993) 379 [hep-th/9207049] [INSPIRE]. 
[42] S. Stieberger, D. Jungnickel, J. Lauer and M. Spalinski, Yukawa couplings for bosonic Z $(N)$ orbifolds: their moduli and twisted sector dependence, Mod. Phys. Lett. A 7 (1992) 3059 [hep-th/9204037] [INSPIRE].

[43] M. Bershadsky and A. Radul, Conformal field theories with additional $Z(N)$ symmetry, Int. J. Mod. Phys. A 2 (1987) 165 [inSPIRE].

[44] J.J. Atick, L.J. Dixon, P.A. Griffin and D. Nemeschansky, Multiloop twist field correlation functions for $Z(N)$ orbifolds, Nucl. Phys. B 298 (1988) 1 [INSPIRE].

[45] T.T. Burwick, R.K. Kaiser and H.F. Muller, General Yukawa couplings of strings on $Z(N)$ orbifolds, Nucl. Phys. B 355 (1991) 689 [inSPIRE].

[46] K.-S. Choi and T. Kobayashi, Higher order couplings from heterotic orbifold theory, Nucl. Phys. B 797 (2008) 295 [arXiv:0711.4894] [INSPIRE].

[47] O. Lebedev et al., The heterotic road to the MSSM with R parity, Phys. Rev. D 77 (2008) 046013 [arXiv:0708.2691] [INSPIRE].

[48] H.P. Nilles, S. Ramos-Sanchez, P.K. Vaudrevange and A. Wingerter, The orbifolder: a tool to study the low energy effective theory of heterotic orbifolds, Comput. Phys. Commun. 183 (2012) 1363 [arXiv:1110.5229] [INSPIRE].

[49] J. Schmidt, Local grand unification in the heterotic landscape, Fortsch. Phys. 58 (2010) 3 [arXiv: 0906.5501] [INSPIRE].

[50] F. Brummer, R. Kappl, M. Ratz and K. Schmidt-Hoberg, Approximate R-symmetries and the mu term, JHEP 04 (2010) 006 [arXiv: 1003.0084] [INSPIRE].

[51] L.J. Dixon, V. Kaplunovsky and J. Louis, On effective field theories describing $(2,2)$ vacua of the heterotic string, Nucl. Phys. B 329 (1990) 27 [InSPIRE].

[52] T. Araki et al., (Non-)abelian discrete anomalies, Nucl. Phys. B 805 (2008) 124 [arXiv: 0805.0207] [INSPIRE].

[53] M. Blaszczyk, N.G. Cabo Bizet, H.P. Nilles and F. Ruhle, A perfect match of MSSM-like orbifold and resolution models via anomalies, JHEP 10 (2011) 117 [arXiv:1108.0667] [INSPIRE].

[54] C. Lüdeling, F. Ruehle and C. Wieck, Non-universal anomalies in heterotic string constructions, Phys. Rev. D 85 (2012) 106010 [arXiv:1203.5789] [INSPIRE]. 